https://doi.org/10.15407/ujpe65.10.835

YU.P. STEPANOVSKY

National Science Center "Kharkiv Institute of Physics and Technology"

(1, Akademichna Str., Kharkiv 61108, Ukraine; e-mail: yustep@kipt.kharkov.ua)

\title{
SQUARE ROOTS OF KEPLER ELLIPSES, ELECTRONS AND RYDBERG ATOMS IN A MAGNETIC FIELD
}

Young Kepler's daring ideas on the structure of the Solar system are applied to the analysis of planetary distances in the exoplanetary system HD 10180. Using Zhukovsky's transformation, the essence of the spinor regularization of Kepler's problem is explained as extracting the square root of an ellipse and using a Kepler eccentric anomaly instead of the usual time. The achievements of Kharkiv radio astronomers in the search for radio recombination lines of Rydberg carbon atoms at the UTR-2 radio telescope are considered. A generalized spinor regularization of the Kepler problem is used to analyze the energy spectra of Rydberg hydrogen atoms in a magnetic field.

Ke ywords: planets, Kepler problem, Rydberg atoms, magnetic field.

\begin{abstract}
Le nez de Cléopâtre: s'il eût été plus court, tout la face de la terre aurait changé.

Blaise PASCAL, 1669

Cleopatra's nose: if it had been shorter, the whole face of the Earth would have changed.
\end{abstract}

Blaise PASCAL, 1669

\section{Introduction}

Science and education in Ukraine are going through a really tough time. The prophecy of the German physicist and philosopher Georg Christoph Lichtenberg (1742-1799) has come true: "Nowadays we everywhere seek to propagate wisdom: who knows whether in a couple of centuries there may not exist universities for restoring the old ignorance." [1] Certainly, G.C. Lichtenberg was writing about European Universities. But here is an opinion of Piotr Nowak, a philosophy professor of Białystok University, about a reformation of today's Polish Universities taken from his book with the expressive title "Nurturing troglodytes" [2]: "There are at least two latent goals

(c) YU.P. STEPANOVSKY, 2020

ISSN 2071-0194. Ukr. J. Phys. 2020. Vol. 65, No. 10 of the University reforms. The first is to divert young and talented youth from intellectual work, from reading and writing books, and teaching for the benefit of quasibusiness activity, which is reduced to collecting money for an enterprise earlier known as University. The second goal, a more demonic one, which agrees with the trend of global changes, is to consistently undermine the instinct of freedom, which means to create definite conditions to exchange academic freedom (and freedom in general) for material welfare". In September 2000, an outstanding mathematician V.I. Arnold speaking at the Dubna conference was very optimistic: "We ${ }^{1}$, are lagging behind the progressive societies as usual. Everywhere science and culture are being destroyed, more slowly, though, in this country than elsewhere, and this may mean that there still is some hope that we could preserve our established cultural level longer than the so-called more developed countries" [3]. It looks like we have

\footnotetext{
${ }^{1}$ Apparently, with the word "we" V.I. Arnold referred not only to Russia, but also to all post-Soviet space.
} 
managed to catch up with "progressive societies", and we are even ahead of them in some innovations. A bit of good news is that this brought us to the community of "progressive societies", the status we have long been dreaming about.

We may be even more encouraged, if we turn to the history of science and history as a whole, which reconcile us "with imperfection of apparent course of events as with common phenomenon in all centuries" [4].

In January 1610, aiming a self-made optical telescope into the sky, Galileo Galilei saw many new and extremely interesting objects including Jupiter's four satellites (Galileo called them planets, but later on, his friend Johannes Kepler called them satellites). In his letter to Johannes Kepler (in una lettera a Giovanni (!) Keplero) of August, 19, 1610 [5], Galileo complains about the most prominent Padua professors who "despite being repeatedly invited, were reluctant to look neither at the planets, nor at the Moon, nor at the telescope". Galileo is asking Kepler, "What is to be done? Whose side to take, Democritus' or Heraclitus'?" (Quid igitur agendum? cum Democrito aut cum Heraclito standum?), and decides to support Democtritus": "My dear Kepler, let us laugh at a great human stupidity!" (Volo, mi Keplere, ut rideamus insignem vulgi stultitiam!)

Galileo continued deriding "the human stupidity". In 1623 he wrote [6]: “Concerning the Copernicus' hypothesis, we, Catholics, are happy to be salvaged from delusion and healed from blindness by the heavenly wisdom". Everybody knows what his derision resulted in: the 1633 inquisition trial, the most disgraceful case in the history of the Catholic Church, which impeded the development of Italian science for a long time. Still we can also find some relief: eventually, the Italian science revived, which is strong evidence that destructive capacities of human stupidity are luckily not unlimited.

What is to follow. Section 2 will feature the life stories of Tycho Brahe and Kepler, which will inevitably make us Heraclite's supporters. Section 3 will show how almost insane ideas of 25-year-old Kepler about the structure of the Solar system can be applied to the exoplanetary system discovered in 2010 and located at a distance of 127 light years from the Solar system. In Section 4, we will find a square root out

\footnotetext{
${ }^{2}$ Unlike Democritus, Heraclitus thought that human stupidity should be weeped for.
}

of an ellipse and learn that it is also an ellipse. It is called a "spinor regularization of the Kepler problem," and it simplifies this problem greatly. In Section 5, we will discuss the achievements of Kharkiv radio astronomers in the search for the radiolines of Rydberg carbon atoms using an UTR-2 radio telescope. The last Section 6 will be devoted to properties of the movement of hydrogen atoms in a stable homogeneous magnetic field, in Fock-Darwin quantum dots, and under quantum Hall effect. We will also analyze the energy spectra of Rydberg hydrogen atoms in a magnetic field using a generalization of the spinor regularization of the Kepler problem, when the square root is found not of planets' orbits but rather of classical orbits of highly excited electrons. In the Appendix, we will discuss several problems pertaining to the innovations following the recent (2019) radical reform of the SI system.

\section{Tycho Brahe and Johannes Kepler}

Unlike Cleopatra, whose nose was capable of changing the face of the Earth, the famous Danish astronomer Tycho Brahe did not have a nose at all ${ }^{3}$. Actually, Tycho did have a nose until he was 22 , but in 1567 a student Tycho Brahe lost it after a quarrel with his friend caused by different viewpoints on some mathematical problem. After a sword duel, Tycho Brahe had to wear an artificial nose made of wax (or a gold and silver alloy), which was quite uncomfortable. The loss of his nose, though, provided him with a lucky opportunity to give up any aristocratic conventionalities and completely devote himself to astronomy, which was considered disgraceful, at that time, for a respected person from a noble family.

Frederick II, the then king of Denmark, duly favored Brahe's noble family. In June 1565, when crossing the bridge, the king suddenly fell from his horse into the cold water. The king could not swim and started to drown. The admiral Jørgen Brahe plunged into the river, saved the king, but in a few days died of pneumonia. Tycho Brahe was thought to be the admiral's son. In fact, he was Jørgen Brahe's nephew, but when Tycho was about one year old, his uncle, who was childless but really wealthy, kidnapped the child from his brother Otto and adopted Tycho. After his uncle's death, Tycho acquired a big

\footnotetext{
3 One can find quite a few sad facts about Tycho Brahe and Johannes Kepler in the books [7-10].
}

ISSN 2071-0194. Ukr. J. Phys. 2020. Vol. 65, No. 10 
legacy and went on to study in European Universities. In 1571, Tycho's own father died and his wealth increased. This is all worth noting, because Tycho Brahe spent almost all his fortune on important astronomical research. (In the same year of 1571, Johannes Kepler was born. And 29 years later in Prague, the fate - remember Cleopatra's nose! - brought Tycho Brahe and Kepler together for a short while, which caused groundbreaking changes to the face of astronomy, because it was Kepler who was destined to make Tycho Brahe's priceless scientific legacy everlasting.)

In 1576, the King of Denmark Frederick II, who valued sciences and arts, found out that Tycho Brahe was going to permanently move to Germany and build an observatory there. The King immediately summoned Tycho Brahe from Germany and he made an effort to persuade Tycho to stay in Denmark. In addition, Frederick II granted him the island of Hven and helped to build on it the Uraniborg observatory, the best one at that time. Over 20 years Tycho worked hard making numerous astronomical observations, which extreme accuracy was incredible given the fact that the optical telescope had not yet been invented. Interestingly, Frederick II contributed 100,000 thalers for Uraniborg's construction, almost as much as Tycho Brahe did. Actually, 100,000 thalers was a really big sum of money. For instance, at the end of the 16th century, Johannes Kepler, then the teacher at a protestant school in Graz, was paid 200 guldens ( $\simeq 171$ thalers) and Galileo Galilei, the professor of Padua University, earned 180 florines ( $\simeq 180$ thalers $)^{4}$.

After the death of Frederick II, his son Christian IV became the King of Denmark and reigned for 59 years. Christian IV was notable for his courage and wit, he waged wars, developed the trade, built towns and ships, but he didn't have the slightest idea why he, and Denmark in general, needed astronomy and proud, independent, and arrogant Tycho Brahe. The King forbade Tycho Brahe to continue his observations and research. He deprived Tycho Brahe of the

\footnotetext{
4 In 1599, a year before Giordano Bruno was burned in Rome, the Venetian senate received a denunciation in respect of immorality of professor Galileo, who lived together with an unmarried Venetian woman. The verdict of the Venetian senate astonished the informant: taking into account the newly discovered facts, professor Galileo should not be paid 180 florins, but rather twice as much, i.e. 360 florins [11].
}

island of Hven, the observatory, and the estates which had been granted by Frederick II. Deeply offended, Tycho Brahe sold out everything still left, and on April, 29, 1597, he abandoned Uraniborg and the island of Hven never to return. In spring of 1599, after long wanderings, Tycho Brahe reached Prague, the capital of the Holy Roman Empire, where the Emperor Rudolf II welcomed him with royal honors. Unfortunately, Tycho Brahe did not live in Prague for a long time. On October, 13, 1601, during a lengthy and lavish dinner with a noble party, "he put politeness before his health" (as noted by J. Kepler in the Diary of Observations kept by Tycho Brahe). As a result, Tycho Brahe developed a severe ischuria ${ }^{5}$ and a terrible fever caused by it. "On October 24, his delirium ceased for several hours; nature conquered and he expired peacefully among the consolations, prayers and tears of his people. So from this date, the series of celestial observations was interrupted, and his own observations of thirty-eight years have come to the end", this was Kepler's final note in Tycho Brahe's Diary of Observations [12].

After Tycho Brahe's death, the invaluable scientific treasures - the results of all of his observations - appeared in possession of Johannes Kepler, who was invited to Prague by Tycho Brahe as his assistant. In 1597, Kepler sent his book Mysterium cosmographicum (The secret of the world) [13] to Tycho Brahe and Galileo Galilei, thereby introducing himself to them. J. Kepler did a tremendous work thoroughly analyzing Tycho Brahe's long-term observations over the planet Mars. It is this analysis that brought Kepler to a discovery of the three laws of planetary motion. In 1607 in Heidelberg, Kepler published Astronomia nova (New astronomy) [14], where he formulated two laws of planetary motion:

I. A planet moves in an elliptic orbit, having the Sun as one of the foci.

II. A straight line joining the planet to the Sun sweeps out equal areas in equal lengths of time.

In 1619, Harmonices Mundi (Harmony of the world) [15] was published by Kepler. It featured Kepler's third law:

III. The squares of the orbital periods of the planets are directly proportional to the cubes of the major axes of their elliptic orbits.

${ }^{5}$ Ischuria, from ancient Greek $\iota \sigma \chi \omega$ ov $\rho \circ \nu,-$ urine retention. 
Incredible assiduity and conditions Kepler had to work in are really impressive. The calculations that helped Kepler to discover the third law occupied seven thick volumes. Each calculation about 10 pages long was reproduced 70 times [16]. This hard labor was performed by a person with poor health and poor sight, who had to compose endless calendars and horoscopes to feed his family. After Tycho Brahe's death, J. Kepler was appointed the Emperor's mathematician at the court of the Emperor Rudolf II with annual salary of 500 guldens. During his life, Kepler saw three Emperors, all of whom truly respected Kepler. What he also saw was that the Emperor's treasury was always empty. The Emperor's 30-year debt to Kepler amounted to 12,694 guldens. In addition, J. Kepler spent several years of his life proving his mother's innocence, who was accused of being a witch and sorceress. Kepler lived his final two years in the town of Sagan serving as Herzog von Wallenstein's court astrologist. In autumn 1630, astride his old worn-out nag, he headed from Sagan (now Żagań, Poland) through Nuremberg to the town of Regensburg in order to find the Emperor Ferdinand II there and once again remind him of the long-standing debt. Kepler did not find the Emperor, but, having ridden $600 \mathrm{~km}$, he caught a cold on his way and on November, 15, 1630, he died leaving his wife and four little children in complete poverty. Kepler had sold his old weary nag for 2 florins [10].

In a 1930 article devoted to the 300th anniversary of Johannes Kepler's death, Albert Einstein wrote on Kepler's discovery of the three laws of planetary motion [17]: "Our admiration for this splendid man is accompanied by another feeling of admiration and reverence, the object of which is no man but the mysterious harmony of nature into which we are born. The ancients already devised the lines exhibiting the simplest conceivable form of regularity. Among these, next to the straight line and the circle, the most important were the ellipse and the hyperbola. We see the last two embodied - at least very nearly so - in the orbits of the heavenly bodies.

It seems that the human mind has first to construct forms independently before we can find them in things. Kepler's marvelous achievement is a particularly fine example of the truth that knowledge cannot spring from experience alone but only from the comparison of the inventions of the intellect with observed fact".

\section{Kepler's Mysterium Cosmographicum and Exoplanetary System HD 10180}

Wolfgang Pauli (1900-1958) met Carl Gustav Jung (1875-1961) in 1930. Their friendly relations and personal correspondence would last until Pauli's death [18]. This friendship significantly enriched the outlook of them both: Jung started to write about photons and wave-particle duality, while Pauli, in turn, got interested in Jung's archetype theory and psychology of scientific creativity. This explained Pauli's keen interest in the personality of J. Kepler. In March 1950, Pauli delivered the lecture "The Influence of Archetypal Ideas on the Scientific Theories of Kepler" before a small audience in Princeton. Abraham Pais attended that lecture [19]. The only thing Pais remembered about the event was that Einstein was also present there but fell asleep to the sound of Pauli's words ${ }^{6}$. Pauli explained in his lecture ${ }^{7}$, that " $K e$ pler's three famous laws of planetary motion, upon which Newton based his theory of gravitation (1687), were not what he was originally seeking. A true spiritual descendant of the Pythagoreans, he was fascinated by the old idea of the music of the spheres and was always trying to find harmonious proportions, in which for him all beauty lay. He attached the utmost importance to the geometric claiming that its theorems "have been in the spirit of God since eternity". His basic principle was "Geometria est archetypus pulchritudinis mundi" (Geometry is the archetype of the beauty of the world)." Pauli also reported that, according to Kepler, "the image of the triune God is in the spherical surface ... the human mind bears the same relation to the perfect Divine Mind as does the circle to the sphere ... This picture of the relationship between the human mind and the Mind Divine fits very well with the interpretation of knowledge, already touched upon, as a "matching" of

${ }^{6}$ It was difficult not to fall asleep during that lecture or remember its ideas. The participants appeared in a quandary of Pavlov's dog, which was taught to wag his tail or run away with his tail between his legs depending on the kind of ellipse he saw: vertical or horizontal. But when it saw a circle, the dog immediately fell asleep.

7 Pauli gave the same lecture twice more, in February and March 1948, at the Society of Psychologists in Zurich (see [18]). The lecture was based on his rather large, later published essay [20].

ISSN 2071-0194. Ukr. J. Phys. 2020. Vol. 65, No. 10 
external impressions with pre-existent inner images", i.e. archetypes.

To support this idea, Pauli cited Kepler: "To know is to compare that which is externally perceived with inner ideas (externum sensile cum ideis internis conferre) and to judge that it agrees with them". The understanding of the inner ideas on the arrangement of the Solar system and the World as a whole as devised by the God was the major goal of Kepler's life. He believed that to some extent he had succeeded.

As a new teacher of mathematics and astronomy, the 23-year-old Kepler appeared in a protestant school in Graz in 1594. Kepler was deeply convinced that implicit harmony of the world existed and he was passionate about Copernicus' study not yet forbidden. Kepler tried to figure out the geometric structure of the Solar system. The insight that dawned on him on July, 19, 1595 was overwhelming: the number of planets in the Solar system is six, (other planets were unknown at that time), the number of spaces between them is five, the number of regular polyhedra, the perfect Platonian bodies, is also only five! The God, a great geometrician, could not help but use this coincidence when he created the Solar system! Kepler started a long and thorough work that resulted in publishing his book Mysterium cosmographicum in Tubingen in 1596. In this book, Kepler provided a detailed explanation of his theory, whose main idea was formulated as follows:

"Earth is the measure of all orbits. Let us circumscribe it with a dodecahedron. A sphere circumscribed around the dodecahedron is the sphere of Mars. Let us circumscribe the sphere of Mars with a tetrahedron. A sphere circumscribed around the tetrahedron is the sphere of Jupiter. Let us circumscribe the sphere of Jupiter with a cube. A sphere circumscribed around the cube is the sphere of Saturn. Let us inscribe an icosahedron into the sphere of Earth. A sphere inscribed in it is a sphere of Venus. Let us inscribe an octahedron in the sphere of Venus. A sphere inscribed in it is the sphere of Mercury".

According to Kepler's calculations,

$R$ Earth $/ R$ Venus $=R$ Mars $/ R$ Earth $=$

$=\sqrt{3} \times \sqrt{5-2 \sqrt{5}}=1.258409$,

$R$ Venus $/ R$ Mercury $=R$ Saturn $/ R$ Jupiter $=$

$=\sqrt{3}=1.732051$,

ISSN 2071-0194. Ukr. J. Phys. 2020. Vol. 65, No. 10
$R$ Jupiter $/ R$ Mars $=3$.

Kepler was lucky. Immediately evident was some agreement with parameters of planetary orbits, which Kepler found in Nicolaus Copernicus' book "On the revolution of heavenly spheres" (1543). To improve the agreement, Kepler had to add some "thickness" to the geometric spheres, in which he inscribed the regular polyhedra. Kepler's model was really imperfect, with the nature implying that the planets did not move in circular orbits and the Sun was not the center of the planetary orbits. This was just the beginning of Kepler's hard way to the discovery of the three laws, the most important laws for the development of celestial mechanics, which eventually got his name.

Approximate agreement of Kepler's model with reality can be explained by the fact that the distances from the planets to the Sun and the planets' orbital periods are known [21] to approximately obey geometric progression with definite coefficients. In addition, the success of Kepler's model resulted from the fact that it was true for the Solar system and for the time Kepler lived at. We will later see that, in another exoplanetary system, where there is no life and there was no Kepler, the law of geometric progression does work, while Kepler's model does not, since the geometric progression coefficients are different there.

Kepler realized that the relation of the radii of circumscribed and inscribed spheres are equal for an icosahedron and a dodecahedron (1.258), for a cube and an octahedron (1.732), while, for a tetrahedron, this relation is the biggest (3). The relations of distances between the planetary orbits are expressed as five numbers. One of them, the biggest, is nearly 3, two are almost twice smaller than 3 , and the other two are still smaller. It became clear which polyhedron to place into which space and what "thicknesses" to add to immaterial spheres (in 1596 Kepler was not aware that the planetary orbits have "aphelia" and "perihelia"). If Kepler had known about the existence of other planets, he would have easily seen the mathematical construction of the whole Solar system. He would have used just two icosahedra and seven cubes:

Mercury - CUBE - Venus - ICOSAHEDRON Earth - ICOSAHEDRON - Mars - CUBE Ceres - CUBE - Jupiter - CUBE - Saturn $C U B E$ - Uranus - CUBE - Neptune - CUBE Ultima Thule. 


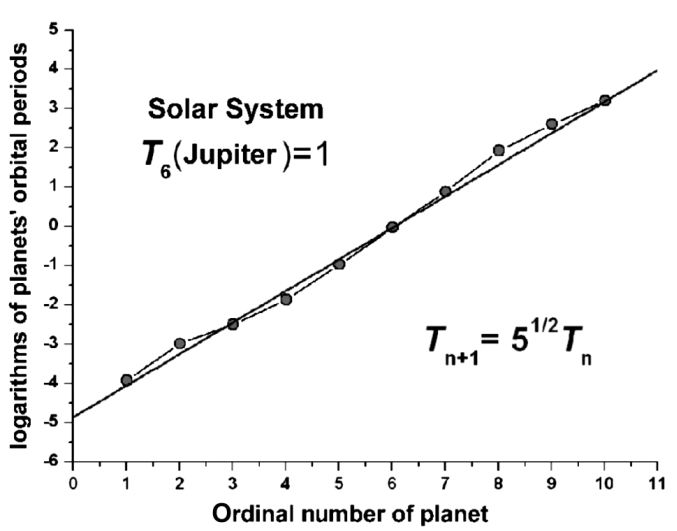

Fig. 1. Deviation of the Solar system planets' orbital periods from a geometric progression

We took a dwarf planet Ceres as a specimen of the asteroid belt between Mars and Jupiter. The transNeptunian Kuiper belt is presented not by a dwarf planet Pluto, but rather a trans-Neptunian object (486958) 2014 MU69, which got the name of Ultima Thule $^{8}$. It seems more natural, since Ultima Thule is an example of the so-called "classical Kuiper belt objects", trans-Neptunian objects that move along almost circular orbits and have no resonance with Neptune, unlike Pluto, one of the "resonance Kuiper belt objects".

According to the above-mentioned pattern "... $a$ planet - CUBE - a planet - CUBE - ...", interplanetary distances approximately follow a geometric progression with a coefficient $\sqrt{3} \approx 1.732$. Together with Kepler, let us imagine that the God, a great geometrician, could not but notice such a mathematical pearl as "the golden ratio". When creating the Solar system, he might have rationalized like this: the orbital periods of neighboring planets should be defined by the "best" irrational number, so that adjacent planets could not interfere with each other's movement due to the resonances. Apparently, the "best" irrational number is "the golden ratio," since the chain fraction

$\Phi=\frac{\sqrt{5}+1}{2}=1+\frac{1}{1+\frac{1}{1+\frac{1}{1+\frac{1}{1+\ldots}}}} \approx 1.618$

contains only ones. However, these planets would have appeared cramped as the relation of their dis-

8 On January, 1, 2019, New horizons spacecraft passed Ultima Thule at a predetermined proximity of $3.500 \mathrm{~km}$ tances would have equaled only 1.378 . Still there is one more "decent" irrational number $\sqrt{5}=2 \Phi-1 \approx$ $\approx 2.236$, it would be perfect for creating a planetary system. In addition, $(\sqrt{5})^{2 / 3} \approx 1.710 \approx \sqrt{3}$, which is also good, because then the interplanetary distances would follow the geometric progression with the coefficient defined by the pattern "- $\boldsymbol{a}$ planet $-C U B E-$ a planet - CUBE". The all-knowing God knew that there was no point in making too accurate calculations, as, with time, all this beauty would be marred due to various unpredictable physical effects and the incredibly difficult mathematical theory of planetary motion.

Let us look now how the real Solar system diverges from regulations of the simplest system (the straight line denotes the geometric progression) (Fig. 1).

The chart for distance logarithms looks quite similar [21]. The real interplanetary distances are approximated well by the geometric progression with the coefficient $\sqrt{3}=1.732 \approx(\sqrt{5})^{2 / 3}=1.710$. As far back as 1913, it was proved by the astronomer Blagg [22].

The history of Kepler's Solar system model is specifically worth reminding today, as over 4 thousand exoplanets ${ }^{9}$ have already been discovered in our galaxy.

The year of 2010 saw the discovery of 7 exoplanets in the Southern Hydra constellation 127 light years away from us. They orbit the yellow dwarf HD 10180, almost as heavy as the Sun $\left(1.06 M_{\odot}\right)$. The orbital period of the first exoplanet with a mass of $1.5 M_{\oplus}$ is 1.18 days, while that of the last one, the seventh, with a mass of $67 M_{\oplus}$, is 2150 days (Fig. 2). This exoplanetary system is 7.3 bn years old.

Let us look how HD 10180 exoplanets' orbital periods deviate from the geometric progression with the coefficient $\frac{3}{2} \sqrt{5}$ (Fig. 2):

It is clearly seen that the deviation of exoplanets' orbital periods from the geometric progression is quite similar to the corresponding deviation for the Solar system.

Apparently, $\frac{3}{2} \sqrt{5}$ is a really nice irrational number, thus Kepler's God had chosen quite a decent coefficient of the geometric progression. The coefficient for planets' distance relation of $\left(\frac{3}{2} \sqrt{5}\right)^{2 / 3} \approx 2.241 \approx$ $\approx \sqrt{5}=2.236$, seems to be His joke: the progression for distances has absolutely no need in such a

${ }^{9}$ In 2019 Michel Mayor and Didier Queloz were awarded the Nobel Prize for the discovery of the first exoplanet in 1995.

ISSN 2071-0194. Ukr. J. Phys. 2020. Vol. 65, No. 10 
nice irrationality as $\sqrt{5}$. Nonetheless, God's joke of granting Kepler the idea of the five polyhedra was really ingenious. By the way, Kepler was so keen on his God that he considered God's occasional banters absolutely natural. In his essay [20], Pauli is citing Kepler's words: "Now, as the Creator played, so he also taught Nature, as His image, to play; and to play the very same game that He played for her first ...".

\section{Square Root of Ellipse or Spinor Regularization of Kepler's Problem}

Before finding a square root of an ellipse, let us discuss some properties of ellipses squared.

To commemorate the 300th anniversary of Newton's Principia, Arnold proved the following theorem [23]:

Any ellipse with focus $O$ is the square of (unique) ellipse with center 0 .

This theorem relates to two problems solved by Newton [24]:

\section{Propositum X. Problem V}

A body revolves in an ellipse; we need to find the law of the centripetal force directed to the center of the ellipse.

Answer:the force is proportional to the distance between the body and the center of the ellipse.

\section{Propositum XI. Problem VI}

A body revolves in an ellipse; we need to find the law of the centripetal force directed to the focus of the ellipse.

Answer: the force is inversely proportional to the distance from the body to the focus of the ellipse.

According to Arnold, the second ellipse is the square of the first one, or Newton's ellipse is the square of Hooke's ellipse ${ }^{10}$. So, in Fig. 3 , the smaller ellipse is the square of the larger one. The semi-major axis of the larger ellipse in the figure equals 1 .

Not only ellipse can be squared. Any aggregate of points, any contour on the plane can be raised to any positive or negative power, if this plane is considered a plane of the complex variable $z=x+i y$.

\footnotetext{
10 Arnold calls the first ellipse Hooke's ellipse and the second one Newton's ellipse because Hooke was the first to suggest that when the force is proportional to the distance, the body revolves in ellipse. We will call the second ellipse Kepler's ellipse or Kepler-Newton's ellipse.
}

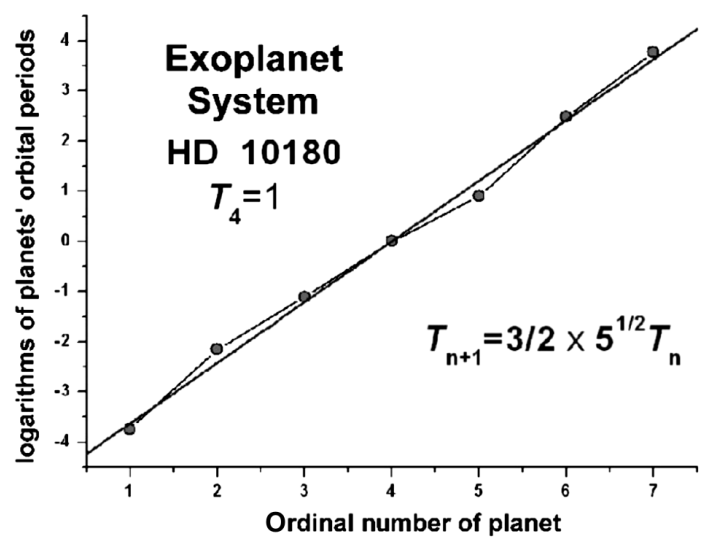

Fig. 2. Deviation of orbital periods of the Exoplanets' HD 10180 from the geometric progression

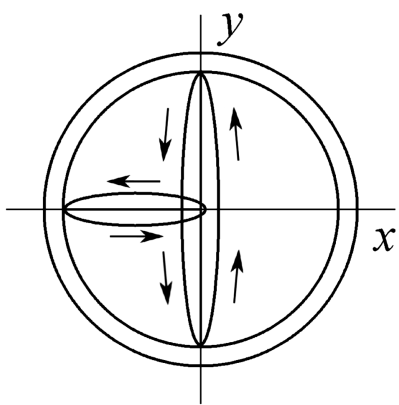

Fig. 3. The smaller ellipse is the square of the larger one

In a simpler case where the eccentricities of both ellipses in Fig. 3 equal one, both Newtonian bodies move back and forth along a segment of a straight line. Let the first body be Lewis Carroll's Alice from Wonderland, who falls through a well dug across the entire globe and, in $2 \pi \sqrt{\frac{R}{g}}=84$ minutes, returns back (the well is the thick vertical diameter of the circle in Fig. 3, $R$ is the radius of the globe, $g$ is the acceleration of gravity). Note that, in the same period of time, "Alice squared" (the thick horizontal radius) will reach the center of the globe and return back twice.

Knowing the time in which Alice returns, we can easily find, e.g., the orbital period of the spacecraft Vostok (89 minutes), which took Yuriy Gagarin to fly round the Earth on April, 12, 1962. In Fig. 3, the radius of the smaller circle equals 1 , the radius of the bigger one is 1.134. Vostok's perigee was 1.027, the apogee was 1.051. That is the semi-major axis of the flight ellipse was 1.039. According to Kepler's third law, Gagarin's flight along the elliptic orbit lasted 
$84 \times \sqrt{1.039^{3}}=89$ minutes. (We used the statement that "Newton's apple" launched at the orbital velocity around an atmosphereless Earth in a circle of radius 1 would return in the same time as Alice does. The oblate shape of the Earth can be neglected as the ratio of the equatorial radius of the Earth and its polar radius equals 1.0034 .)

In Fig. 3, the bigger ellipse is a circle flattened 8 times. Since Arnold's theorem implies that the center of the circle becomes the focus of the smaller ellipse, the perihelion of this ellipse equals $1 / 64$, hence the aphelion of this ellipse is 64 times greater than the perihelion.

Note that the flattening of the smaller ellipse is almost equal to that of Halley's comet, where aphelion (1948) - perihelion (1986) ratio equals 60 [25]. The appearance of Halley's Comet in 1682 was crucial for the development of celestial mechanics and science as a whole. It is generally known that Newton would have never written his Principia, if Halley, who became interested in the comet's motion, had not made Newton do it. In early 1684, Edmond Halley traveled from London to Cambridge to ask Newton if the force inversely proportional to square of the distance to the Sun would make the comet move in an ellipse. What happened next is well known [26]. Newton answered that it would, as he calculated that 20 years earlier. Still Newton could not show his calculations to the astonished Halley, as he failed to find them. But after Halley left, he found the calculations and, on encountering a minor mistake, reproduced them in a while. In November 1684, Newton sent Halley a small nine-page treatise De Motu Corporum in Gyrum (On the motion of a body in an orbit). Halley later would say that "he was the Ulysses who produced this Achilles" "11. Newton abandoned his alchemical, chronological, and theological research and got down to Principia. He forgot about sleeping, eating, and everything else. In 18 months of the painstaking work, which almost ruined Newton, Principia was finished, Newton's Troy had fallen. The publishing of Principia had to be paid by Halley as the Royal society appeared to be short on money for that.

\footnotetext{
${ }^{11}$ Ulysses (Odysseus) encouraged Achilles to attack Troy for he knew that Achilles was the only person to defeat the Troyans. Similarly, Halley urged Newton to write Principia. Achilles perished on the eve of Troy's fall; creating Principia caused Newton's complete physical and mental exhaustion.
}

Based on Newton's Principia, Halley calculated the orbital elements of 24 comets (from 1337 till 1698) and noticed a remarkable similarity of the three comets seen in 1531, 1607 (observed and described by Kepler) and 1682. All three comets had the same perihelia $(0.58 \mathrm{au})$ and orbital inclinations $\left(1620^{\circ}\right)$ [27]. Halley reasonably assumed that all the three comets were one and the same comet and predicted that it would return in 1758 , which appeared true. Since then it has been righteously called after Halley.

The property of two ellipses lying in the same plane, one being the square of the other (Arnold's theorem), was discovered by Levi-Civita [28] in 1903. However, Halley's comet's orbital inclination $\left(1620^{\circ}\right)$ to the ecliptic plane (which for all planets could be well approximated to our complex variable plane) is significantly different from zero. Then is it possible to find a square root of its ellipse, or any ellipse, in threedimensional space? As it was proved in 1964, it is really possible [29]. To find a square root in the general case, astronomers had to rediscover spinors. Since the transition from conventional coordinates to spinors reduces a rather complex Kepler's problem on the motion in the singular potential $U=-1 / r$ to $\boldsymbol{a}$ much simpler problem on the motion in the potential $U=r^{2}$, such transition is called the spinor regularization of Kepler's problem.

Let us return to Newton-Arnold ellipses and calculate how the ellipse oblateness changes, when it is squared. If the vertical ellipse in Fig. 3 is flattened $A$ times, then the aphelion of the smaller ellipse is equal to $1 / A^{2}$, i.e. the perihelion of the smaller ellipse is $A^{2}$ times bigger than the aphelion. This relation unambiguously determines how many times $B$ this ellipse is flattened, $B=\frac{1}{2}\left(A+\frac{1}{A}\right)$. In our case, Fig. $3, A=8, B=4 \frac{1}{16}$. Conformal transformation of the complex variable $z^{\prime}=\frac{1}{2}\left(z+\frac{1}{z}\right)$ is called after Zhukovsky, as in 1910 N.Ye. Zhukovsky successfully employed this transformation to calculate the lift force of different aerodynamic profiles, which appear when Zhukovsky transformation acts upon a circle, if the center of the circle does not coincide with the complex number $z=0$ [30]. If the center of the circle coincides with $z=0$, then it could be easlily seen that, under the Zhukovsky transformation $z^{\prime}=\frac{1}{2}\left(z+\frac{1}{z}\right)$, any circle with a radius different from one will turn into an ellipse with the center in the center of the circle, and, under the dual

ISSN 2071-0194. Ukr. J. Phys. 2020. Vol. 65, No. 10 
Zhukovsky transformation $z^{\prime}=\left(\frac{1}{2}\left(z+\frac{1}{z}\right)\right)^{2}$, it will turn into an ellipse with the focus in the center of the circle [23]. Let an arbitrary point on the circle with a radius $r \neq 0$ correspond to the complex number $z=r e^{i \varphi}, 0 \leq \varphi \leq 2 \pi$. The angle $\varphi$ is an extremely important variable (eccentric anomaly $E$ ) in Kepler's theory. Figure 3 shows a circle with the radius $R=\sqrt{\frac{9}{7}}=1.134$, as, under the Zhukovsky transformation $z^{\prime}=\frac{i}{\sqrt{\frac{9}{7}+\frac{7}{9}}}\left(z+\frac{1}{z}\right)$, exactly this circle (or the circle with the radius $R=\sqrt{\frac{7}{9}}=0.882$ )) is flattened 8 times and turns into a bigger ellipse with semi-major axis 1 presented in Fig. 3. The dual Zhukovsky transformation will produce a smaller ellipse in Fig. 3.

Analyzing Kepler's ellipse shown in Fig. 4, we will see that almost everything that we need to find a square root of the ellipse was made by Kepler.

In Fig. 4, $a$ and $b$ are the semi-major and semiminor axes of the ellipse, $\varepsilon$ is the eccentricity, $x$ and $y$ are the axes of the orthogonal coordinate system whose center coincides with the focus of the ellipse. $E$ is the eccentric anomaly. Given that an ellipse is a circle flattened $\sqrt{1-\varepsilon^{2}}$ times, the coordinates of the planet can be easily found:

$x=a(\cos E-\varepsilon), \quad y=a \sqrt{1-\varepsilon^{2}} \sin E$.

According to the Pythagorean theorem, from (1), we obtain

$r^{2}=x^{2}+y^{2}=a^{2}(\cos E-\varepsilon)^{2}+$

$+a^{2}\left(\sqrt{1-\varepsilon^{2}} \sin E\right)^{2}=a^{2}(1-\varepsilon \cos E)^{2}$,

thus

$r=a(1-\varepsilon \cos E)$.

It is readily seen that

$x+i y=a\left(\sqrt{1-\varepsilon} \cos \frac{E}{2}+i \sqrt{1+\varepsilon} \sin \frac{E}{2}\right)^{2}$,

so Kepler's ellipse is the square of the ellipse with the center in the center of the ellipse, which was discovered by Levi-Civita in 1903 [28].

Let us introduce a two-component quantity

$\psi=\left(\begin{array}{l}\psi_{1} \\ \psi_{2}\end{array}\right)=\sqrt{\frac{a}{2}}\left(\begin{array}{l}\sqrt{1-\varepsilon} \cos \frac{E}{2}-i \sqrt{1+\varepsilon} \sin \frac{E}{2} \\ \sqrt{1-\varepsilon} \cos \frac{E}{2}+i \sqrt{1+\varepsilon} \sin \frac{E}{2}\end{array}\right)$.

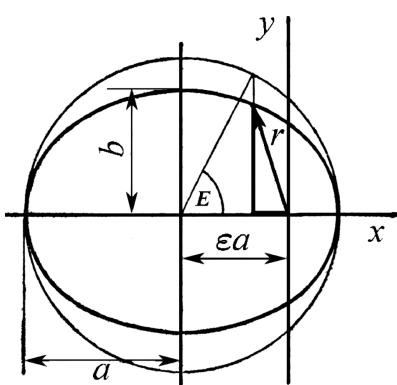

Fig. 4. Kepler's ellipse

We will consider the quantity $\psi$ as a spinor, i.e. assume that when a three-dimensional coordinate system $(x, y, z)$ turns round the axis directed at $\mathbf{e}, \mathbf{e}^{2}=1$, at an angle of $\varphi, \mathbf{e} \varphi \equiv \varphi$,

$$
\begin{aligned}
& \psi \rightarrow \psi^{\prime}=\exp \left(i \frac{\boldsymbol{\varphi} \sigma}{2}\right) \psi \\
& \sigma_{1}=\left(\begin{array}{ll}
0 & 1 \\
1 & 0
\end{array}\right), \quad \sigma_{2}=\left(\begin{array}{cc}
0 & -i \\
i & 0
\end{array}\right), \quad \sigma_{3}=\left(\begin{array}{cc}
1 & 0 \\
0 & -1
\end{array}\right),
\end{aligned}
$$

$\boldsymbol{\sigma}$ are the standard Pauli matrices.

Spinor (4) corresponds to Kepler's ellipse located in the plane $(x, y)$, more precisely, the final point of the vector $\mathbf{r}=\psi^{*} \boldsymbol{\sigma} \psi, 0 \leq E \leq 2 \pi$ runs along Kepler's ellipse. Note that

$\frac{d \psi^{*}}{d E} \psi-\psi^{*} \frac{d \psi}{d E}=0$

Now, we have to ascertain how the eccentric anomaly $E$ relates to the time in which the planet moves in the ellipse. Here, Kepler's second law will help. According to this law, $\frac{d S}{S}=\frac{d t}{T}$, where $S=\pi a b$ is the area of the ellipse, $T$ is the planet's sidereal period. By calculating

$d S=\frac{1}{2}|[\mathbf{r}, d \mathbf{r}]|=\frac{1}{2}|x d y-y d x|=$

$=\frac{1}{2} a^{2} \sqrt{1-\varepsilon^{2}}(1-\varepsilon \cos E) d E$,

we find that

$d t=\frac{T}{2 \pi}(1-\varepsilon \cos E) d E=\frac{T}{2 \pi a} r d E$,

thus

$t=\frac{T}{2 \pi}(E-\varepsilon \sin E)$,

(assume that, when $t=0$, the planet is in the perihelion). Kepler's equation (9) is his most important 
equation in the theory of planets' motion, as well as in the theory of spinor regularization of Kepler's problem. (For more details on spinor regularization of Kepler's problem, see [31, 32].)

\section{Hyperfine Structure}

\section{of a Hydrogen Atom, Rydberg}

\section{Atoms, and UTR-2}

In 1947, the famous astrophysicist of the XX century I.S. Shklovsky accidentally read in the English Observatory magazine about a bold idea of a 23-year-old Dutch student H. van de Hulst. In 1944, the latter suggested that the emission lines of atomic hydrogen with a wavelength of $21 \mathrm{~cm}$ existed in the radio spectrum of the Galaxy. No details were reported. Shklovsky was captivated by this idea. "The very possibility of literally counting all the hydrogen atoms of the interstellar medium, finding the temperature of its clouds, their kinematics and dynamics was fascinating," he wrote in his memoirs [33]. In 1948, Shklovsky calculated the lifetime of the excited level of a hyperfine structure (11.5 million years) and came to the conclusion that a hydrogen radio line with the wavelength of $21 \mathrm{~cm}$ could be observed! To "catch" this wave, Shklovsky found a talented radio astronomer V.V. Vitkevich, who zealously undertook the experimental implementation of the " $21 \mathrm{~cm}$ project". However, in the early 1950s, he suddenly quit the " $21 \mathrm{~cm}$ project without any explanation".

Only 20 years later, Vitkevich told Shklovsky what had happened [33]: Cleopatra's nose intervened in the "21 cm project"! It turned out that Viktor Vitkevich sometimes visited Lev Landau's house (their wives were relatives), where he used to be modestly silent. Still, once he carelessly and lightly told Landau about the "21 cm project". Landau reacted to Vitkevich's story as follows: "Big deal - calculating the probability of magneto-dipole radiation! There are corresponding formulas in my book, and any student can do such calculations. But where did Shklovsky find the density of hydrogen in the interstellar medium? This is pure pathology". Unfortunately, Vitkevich did not receive appropriate instructions from I.Y. Tamm on time, which he gave to everyone who had something to tell Landau [34]: "When he swears - says that it is "philology", "pathology", "nonsense", - ignore it, but as soon as he begins to speak straight to the point - be all ears and take note of it". That is, Landau said nothing special and did not get to the bottom line. Vitkevich's reaction to his words enabled American scientists H. Ewan and E. Purcell to be the first to catch a $21 \mathrm{~cm}$ wave in the spring of 1951 .

As for the book, Landau was right, indeed, on pp. 547-548 of "Quantum Mechanics" published in 1948 [35], the Fermi formula was derived [36],

$\Delta E=\frac{8 \pi \beta_{0} \beta}{3 i}(2 i+1)|\psi(0)|^{2}=\frac{8 \beta_{0} \beta}{3} \frac{(2 i+1)}{a^{3} n^{3} i}$

for the energy splitting $\Delta E$ between two levels of hyperfine structure of $n s$-states of alkaline elements' atoms ${ }^{12}$. In the Landau-Lifshitz approach, the electron magnetic moment is equal to the Bohr magneton $\beta_{0} \equiv \mu_{B}=\frac{e \hbar}{2 m_{e} c}$, but it can be arbitrary, $\beta$ is the nuclear magnetic moment, $i$ is the nuclear spin, $\psi(0)$ is the wave function of the $s$-electron at the origin, $a=\frac{\hbar^{2}}{m_{e} e^{2}}$ is the Bohr radius. It should be explained what $\psi(0)$ is: the electron wave function $\Psi_{\alpha}(\mathbf{r})$ in the Schrödinger-Pauli equation is the spinor, $\alpha=1,2$, for the $n s$-electron, $\Psi_{\alpha}(\mathbf{r})=\psi(r) u_{\alpha}, u^{*} u=1 . \psi(r)$ is the conventional Schrödinger wave function in the absence of a magnetic field (the magnetic field of the nucleus is considered a small perturbation). Using formula (10), the frequency and the wavelength of the radiation of alkaline hydrogen atoms, or deuterium, can indeed be calculated by any student. However it had to be Fermi to derive this formula!

Unlike Landau and Lifshitz, Fermi did not consider the Schrödinger-Pauli equations, but the Dirac equation, that is in Fermi's works the magnetic moment of the electron was fundamentally equal to the Bohr magneton, as it follows from the Dirac equation $^{13}$. Fermi considered the spinor $\Psi_{\alpha}(\mathbf{r})=\psi(r) u_{\alpha}$ as the third and fourth ("large") components of the Dirac wave function, the other two components, the first and second ("small") ones are expressed in two "large" ones, the result can be expressed only in two

12 When Shklovsky was calculating the hyperfine hydrogen splitting in the summer of 1948 , he had no chance to use the formula (10) from Landau and Lifshitz's book, as it did not exit at that time; the textbook was approved for publishing only on July, 9, 1948.

13 "I have used Dirac's theory of the electron, since the simpler Pauli's theory gives the wrong result", wrote Fermi in a short report on his research in Nature [37]. Landau and Lifshitz, though, derived a correct (???) Fermi formula from Pauli's theory

ISSN 2071-0194. Ukr. J. Phys. 2020. Vol. 65, No. 10 
"large" components, and Fermi did so. Fermi rightfully thought that, as, in a non-relativistic approximation, "large" components followed the Schrödinger equation, the Dirac equation and "small" components could be neglected and $\psi(r)$ could be considered as the Schrödinger wave function. Fermi thought it was evident, and it surely is, but it is absolutely different for a hydrogen atom! In 1928, Darwin found out [38] that, for a hydrogen atom, $\psi(0)=\infty$ ! Fermi referred to Darwin's paper in his 1930 work, but he missed the infinity of $\psi(0)$ and brought it up three years later in his 1933 paper [39], writing about formula (10), "Evidently, though, this formula cannot be used in the relativistic case". The coauthor of the paper was Emilio Segré, but it was Ettore Majorana who helped Fermi to deal with infinity of $\psi(0)$.

The infinity of $\psi(0)$ is caused by the fact that the singularity of Kepler's problem in a Coulomb field becomes even greater in the relativistic case (singularity of $1 / r^{2}$ is added to the singularity of $1 / r$ ). However in reality (in nature), there is no singularity and no infinity related to it. Darwin and Fermi considered a point electron in a point proton field. But the proton is not a point-like particle. The way out is to take into account that the proton is not a point-like object. It was first done in 1945 by Pomeranchuk and Smorodinsky [40]; they considered the proton as a uniformly charged sphere with the radius of $1.2 \mathrm{fm}$ ( $1 \mathrm{fm}=10^{-15} \mathrm{~m}$, or fermi, femtometer). The value of the Dirac wave function appeared finite and very close to the values of the Schr'odinger wave function. Fermi's intuition did not betray him ${ }^{14}$.

If we take experimental data of the year 1947 for electron's, proton's. and deuteron's magnetic mo-

\footnotetext{
14 Intuition sometimes did betray even the great Enrico Fermi. Laura Fermi, who was quite successful in fighting Enrico's habit of overusing the word "evidently", recalled [41] that the winter of 1928-1929 in Italy was extremely severe, the temperature in the rooms where the young couple lived did not exceed $+8{ }^{\circ} \mathrm{C}$. Enrico's theoretical instructions on how to stoke the oven wouldn't help. When Laura suggested buying winter window frames, Enrico got down to lengthy calculations and declared that the penetration of cold air through the window frames was so insignificant that winter frames were evidently useless. Several months later, "His Excellency" Enrico (just now Fermi was appointed a member of the Royal Academy of Italy by Mussolini) found a mistake in his calculations: he had put the decimal point in the wrong place and approved buying the frames.
}

ments, then, according to the Fermi formula (10),

$\nu_{H}=1416.90(54) \mathrm{MHz}, \quad \lambda_{H}=21.1583(81) \mathrm{cm}$

$\nu_{D}=326.53(16) \mathrm{MHz}, \quad \lambda_{D}=91.812(44) \mathrm{cm}$.

In 1947, it was found out that the formulas (11) disagreed with the experiments as, according to the calculations of Rabi's group [42],

$\nu_{H}=1421.3(2) \mathrm{MHz}, \quad \lambda_{H}=21.090(3) \mathrm{cm}$

$\nu_{D}=327.37(3) \mathrm{MHz}, \quad \lambda_{D}=91.576(8) \mathrm{cm}$.

The discrepancy between formulae (11) and (12) is explained by the fact that electron's magnetic moment is not equal to Bohr's magneton, as followed from the Dirac theory. It was a considerable discovery, whose significance can be compared with Kepler's discovery of the ellipticity of planetary orbits. This discovery marked the beginning of progressive development of quantum electrodynamics, quantum field theory, and theory of elementary particles.

Soon after, in the same 1947, Foley and Kusch [43] found in their experiments that the electron's magnetic moment equaled

$\mu_{e \text { exper. }}=\mu_{B} \times 1.0012(1)$,

and Schwinger [44] theoretically calculated that

$\mu_{\text {theor. }}=\mu_{B}\left(1+\frac{\alpha}{2 \pi}\right)=\mu_{B} \times 1.001162$,

$\alpha=\frac{e^{2}}{\hbar c} \approx \frac{1}{137}$.

The 2008 measurements of electron's magnetic moment

$\mu_{e \exp }=\mu_{B} \times 1.00115965218073(28)$

agree well with the theoretical calculations [46],

$\mu_{\text {theor }}=\mu_{B} \times 1.00115965218067(81)$.

Since the theoretical calculations contain the fine structure constant $\alpha$, its current experimental value [47] is also worth providing:

$\alpha=\frac{e^{2}}{\hbar c}=\frac{1}{137.035999084(21)}$.

The hyperfine splitting in hydrogen and deuterium atoms is also measured with high accuracy [48]:

$\Delta \nu_{H}=1420.4057517667(9) \mathrm{MHz}$,

$\Delta \nu_{D}=327.384352522(2) \mathrm{MHz}$. 
The Fermi formula (10), given the correct value of electron's magnetic moment and the finiteness of proton's and deuteron's masses, enables calculating only the first five digits in these numbers. To calculate the sixth and seventh significant digits, one should account for the distributions of the electric charge and magnetic moments inside the proton and deuteron, which are determined by proton's quark structure and deuteron's proton-neutron structure [50].

Let us return to Shklovsky's radio-astronomical research. A sudden collapse of "the $21 \mathrm{~cm}$ project" was not Shklovsky's last experience connected with hyperfine transitions. Shklovsky self-calculated ${ }^{15}$ the wave lengths of the transition emission of the $1 s$ term hyperfine structure of not only hydrogen (21.1 $\mathrm{cm})$, but also deuterium $(91.6 \mathrm{~cm})$, nevertheless this wave in the Galaxy radio emissions was never detected in Shklovsky's lifetime. Upon calculating in 1951 [51] the corresponding transition frequencies, Shklovsky assumed the possibility of the existence in Galaxy's radio spectrum of two emission lines of the atomic nitrogen ${ }^{14} N$, whose basic state ${ }^{4} S_{3 / 2}$ is split into three levels of hyperfine structure with the spins $5 / 2,3 / 2$, and $1 / 2$. In 1958 , the transition frequencies $(5 / 2 \rightarrow 3 / 2)$ and $(3 / 2 \rightarrow 1 / 2)$, calculated by Shklovsky, were measured under the Earth conditions [52],

$$
\begin{aligned}
& \nu_{5 / 2 \rightarrow 3 / 2}=26.1275 \pm 0.0005 \mathrm{MHz}, \\
& \nu_{3 / 2 \rightarrow 1 / 2}=15.6772 \pm 0.0005 \mathrm{MHz} .
\end{aligned}
$$

Shklovsky found reliable people who enthusiastically embarked on the task of finding the waves with the frequencies (19) in the Galaxy radio emission. It was a team of Kharkiv radio astronomers from the Institute of Radio Electronics (IRE) of the Ukrainian Academy of Sciences headed by S.Ya. Braude. To find the ${ }^{14} N$ nitrogen radio emission line, in a village of Grakove near Kharkiv, the radio telescope UTR-2 (Ukrainian T-like Radio telescope of the 2nd modification) was built, the world's biggest and best decameter radio wave radio telescope, which has been operating since 1970. On June, 17, 1978, the UTR-2 captured a $26.7 \mathrm{MHz}$ signal from Cassiopeia

\footnotetext{
15 The Fermi formula, unknown to Shklovsky, at that time, could be found not only in Landau and Lifshitz's textbook [35]; in 1949 a book [49] was published in Russian which contained the Fermi formula and discussions on Rabi's team experiments of 1947 [42].
}

A (Cas A) supernova remnants in the constellation Cassiopeia ${ }^{16}$. Kharkiv scientists did a great job and found what they had been searching.

On January, 24, 1980, the Nature published the paper by Konovalenko and Sodin "Neutral ${ }^{14} N$ in the interstellar medium" [54], where the authors reported observing the absorption of radio waves from Cas $A$ at the frequency of $26.13 \pm 0,20 \mathrm{MHz}$. As a reaction to this publication, a paper by Blake, Crutcher, and Watson [55] appeared in Nature on October, 23, 1980, where the authors expressed their skepticism about the interpretation of the origin of the signal observed at the UTR-2. They also supposed that it was an emission recombination line of the highly excited (Rydberg) carbon atom $\mathrm{C} 631 \alpha^{17}$. On November, 12, 1981, Konovalenko and Sodin reported in Nature on the UTR-2 observation of the emission recombination lines of $\mathrm{C} 630 \alpha$ and $\mathrm{C} 640 \alpha$ [56]. Later on, the radio lines of $\mathrm{C} 603 \alpha$, $\mathrm{C} 611 \alpha, \mathrm{C} 621 \alpha, \mathrm{C} 628 \alpha-\mathrm{C} 638 \alpha$, C640 $\alpha, \mathrm{C} 686 \alpha, \mathrm{C} 732 \alpha, \mathrm{C} 790 \beta-\mathrm{C} 802 \beta$ [57] were also observed using the UTR-2.

Let us explain the above-mentioned designation Cn $\alpha$. We will use the Rydberg formula to calculate emission frequencies of the Rydberg carbon atom

$$
\begin{aligned}
& \nu_{n+k \rightarrow n}=\frac{e^{4}}{4 \pi \hbar^{3}} \frac{m_{e} m_{N}}{m_{e}+m_{N}}\left(\frac{1}{n^{2}}-\frac{1}{(n+k)^{2}}\right)= \\
& =\frac{\nu_{0}}{1,000039}\left(\frac{1}{n^{2}}-\frac{1}{(n+k)^{2}}\right),
\end{aligned}
$$

where $\nu_{0}=e^{4} / 4 \pi \hbar^{3}=3.2898419602508(64) \times 10^{15} \mathrm{~Hz}$ is the Rydberg constant, whose accuracy was certainly excessive for Konovalenko and Sodin, but, fortunately, the observations of the UTR-2 were accurate enough to understand emissions of which atoms exactly were observed.

The Rydberg formula enables calculating the atom's emission frequency, when the electron moves from the $n+k$-th level to the $n$-th one. The transition $n+1 \rightarrow n$ for a carbon atom (C Carboneum) is denoted as $\mathrm{Cn} \alpha$, the transition

16 Cassiopeia $A$, a powerful radio source located approximately 11,000 light years away from Earth, the type II supernova remnants, was discovered by radio astronomers in 1948 [53]

17 Rydberg atoms with $n \approx 100-1000$ are formed in the interstellar space due to recombination, i.e. firstly the atom's ionization, and then the capture of the lost electron. Hence, the name - recombination lines.

ISSN 2071-0194. Ukr. J. Phys. 2020. Vol. 65, No. 10 
$n+2 \rightarrow n$ as $\operatorname{Cn} \beta$ etc. According to (20), the frequency of the $\mathrm{C} 630 \alpha$ line equals $26.25025 \mathrm{MHz}$, the $\mathrm{C} 631 \alpha$ line $-26.12574 \mathrm{MHz}$, and the $\mathrm{C} 640 \alpha$ line $25.03982 \mathrm{MHz}$. This was the proof that all the three lines were the recombination radio lines (RRL) of exactly carbon Rydberg atoms.

So, thanks to I.S. Shklovsky, the radio-astronomical team headed by S.Ya. Braude reached the world level, Kharkiv had seen outstanding radio-astronomical discoveries (not only those mentioned; for example, the UTR helped obtain important results on the nature of Jupiter's radio emissions [58]). The research at the UTR-2 is going on, and Ukraine has been still holding the world leadership in decameter radio-astronomical observations. I.S. Shklovsky's contribution to the advances in low-frequency radioastronomy is discussed in O.O. Konovalenko's paper [60]; the recombination radio lines (RRL) of the Rydberg atoms were examined in book [62], which highlights the achievements of Kharkiv scientists.

\section{Rydberg Atoms and Adiabatic}

Invariants, Electrons in a Magnetic Field and Fock-Darwin Quantum Dots, Quantum Hall Effect, Rydberg Hydrogen Atom in a Magnetic Field as an Anharmonic Oscillator

\subsection{Boltzmann adiabatic invariant and Rydberg atoms}

We will start with events happening more that a hundred of years ago. On September, 28, 1913, P. Ehrenfest wrote in a letter to A.F. Ioffe, "Bohr's paper 'Quantum mechanical consequences of Balmer's law' makes me desperate: if Balmer's formula can be derived in such a way, then I have to throw away all the physics (and myself withal)" [63]. Bohr's work drove many people to despair, but Ehrenfest was ready to accept this work more than anybody else. In late 1912, he discovered the rule of quantization of arbitrary periodic motion. The quantization of the angular momentum, which was the basis of Bohr's theory, appeared the simplest conclusion of Ehrenfest's theory. According to this theory, Planck's hypothesis could be generalized as follows: one should quantize Boltzmann's adiabatic invariant $2 \tau \bar{T}$, where $\tau$ is the motion period, $\bar{T}$ is the mean value of the kinetic energy $T$ during the motion period,

$2 \tau \bar{T}=n h$.
Using just several lines of transformations, from Ehrenfest's formula (21) and the virial theorem, one can derive both Planck's quantization of harmonic oscillations and the Bohr-Sommerfeld quantization of arbitrary elliptic orbits in a hydrogen atom (for more details and references, see [32]). In the general case of a homogeneous potential $U(\lambda r)=\lambda^{N} U(r)$, from the virial theorem [64], $\bar{T}=\frac{N}{2} \bar{U}$, we obtain $(\nu=1 / \tau)$ :

$E_{n}=\bar{E}=\bar{T}+\bar{U}=\frac{N+2}{N} \bar{T}=n \frac{N+2}{N} \frac{h \nu}{2}$.

For harmonic oscillations $(N=2)$, we obtain Planck's quantization rule:

$E_{n}=n h \nu$.

For a hydrogen atom $(N=-1)$,

$E_{n}=-n \frac{h \nu}{2}$.

Given that, for Kepler's classical motion of an electron in the potential $U=-e^{2} / r$, the energy is defined just by the revolution frequency, $E^{3}=-\pi^{2} m_{e} e^{4} \nu^{2} / 2$ [64], we obtain the Bohr-Sommerfeld quantization rule for arbitrary elliptic orbits,

$E_{n}=-\frac{e^{4} m_{e}}{2 \hbar^{2}} \frac{1}{n^{2}}$

From this, for the hydrogen atom emission frequencies, we obtain the Rydberg formula (20) (disregarding the proton mass)

$\nu_{n+k \rightarrow n}=\frac{E_{n+k}-E_{n}}{h}=\frac{e^{4} m_{e}}{4 \pi \hbar^{3}}\left(\frac{1}{n^{2}}-\frac{1}{(n+k)^{2}}\right)=$

$=\nu_{0}\left(\frac{1}{n^{2}}-\frac{1}{(n+k)^{2}}\right)$,

$\nu_{0}$ is the Rydberg constant.

When Bohr derived the formula (25) for the case of circular orbits in 1913, there arose a sensible question: if an electron revolves in a circle with the frequency $\nu$, it has to emit electromagnetic waves at frequencies $\nu, 2 \nu, 3 \nu \ldots$ (corresponding classical formulae were found by Schott [65] in 1912), or is it Bohr's atom that emits these waves? The answer is: these waves are emitted by hydrogen atoms only in a classical limit, at $n \gg 1$, i.e. by Rydberg atoms. Indeed, in 2000-2003, the UTR-2 revealed simultaneously two parallel series of decametric carbon lines directed to Cassiopeia A [57], C628 $\alpha-$ C638 $\alpha$ and C790 $\beta-$ C802 $\beta$. 
These lines are of the same 1.2 MHz frequency band due to the fact that the corresponding frequencies follow the relation $\sqrt[3]{2} \times(n(\alpha)+1) \approx n(\beta)+2$, e.g. $\sqrt[3]{2} \times 629=792.5$.

Let us ascertain that, at $n \gg 1$, Rydberg electrons emit waves at the frequencies $\nu, 2 \nu, 3 \nu \ldots$. According to (24) and (25), the electron orbital frequency

$\nu=-\frac{2 E_{n}}{h n}=\frac{e^{4} m_{e}}{2 \pi \hbar^{3}} \frac{1}{n^{3}}$.

According to (25), at $n \gg 1$,

$\nu_{n+k \rightarrow n}=\frac{E_{n+k}-E_{n}}{h} \approx \frac{e^{4} m_{e}}{2 \pi \hbar^{3}} \frac{k}{n^{3}}=k \nu$,

which absolutely agrees with the emission of a classical electric charge moving on a classical orbit.

The fact that Ehrenfest's quantization rule (21) is true for a hydrogen atom does not seem strange, because, as we saw in Section 4, Kepler's motion of an electron in the potential $U \sim 1 / r$ is the "square" of the elliptic motion of the particle in the quadratic (parabolic) potential.

The validity of Ehrenfest's rule in a quasiclassical approximation is proved in quantum mechanics for the case of an arbitrary potential $U(r)$, which has coupled states with $n \gg 1$. There are, though, physical problems of another nature, e.g., studying atomic properties in an external magnetic field. In such cases, Ehrenfest's rule, if not generalized, becomes invalid. It seems important to clarify the cause.

\subsection{Electrons in a magnetic}

\section{field and Fock-Darwin quantum dots}

Ehrenfest's rule does not work in the simplest case of a charge moving on a circle with the frequency $\nu$ in the static magnetic field $H$. Its energy levels are defined by formula (21), $E_{n}=n h \nu 2$. The transition $n \rightarrow n-1$ causes the charge to emit a photon with the frequency $\nu / 2$. This contradicts the fact that, at $n \gg$ $\gg 1$, this frequency is equal to $\nu$. Let us show that it is quite possible to obtain $\boldsymbol{a}$ valid quantization rule for a charge moving in a magnetic field from Ehrenfest's rule, if to move to $\boldsymbol{a}$ rotating coordinate system.

Let us put down the equation for the motion of the charge $-e$ with the mass $m_{e}$, which moves in a plane $(x, y)$ in a magnetic field $H$ with the cyclotron angular frequency $\omega=e H / m_{e} c$, as

$\ddot{z}-i \omega \dot{z}=0$, where $z=x+i y$. In the new coordinate $\operatorname{system}(\tilde{x}, \tilde{y})$ rotating with the Larmor frequency $\omega / 2, \tilde{z}=\tilde{x}+i \tilde{y}=$ $=\exp (-i \omega t / 2) z$, we obtain

$\ddot{\tilde{z}}+(\omega / 2)^{2} \tilde{z}=0$.

Note that the trivial transformation from (29) to (30) reveals an important characteristic of the problem of the motion of a charge in a static magnetic field. The fact that two very simple equations, (29) and (30) are equivalent means the equivalence of the charge motion in a magnetic field and body motion on Hooke's ellipse (see Section 4). Since Hooke's ellipse squared is Kepler's ellipse, we have discovered an interesting similarity: the classical problem of the charge motion in a static homogeneous magnetic field is mathematically equivalent to $\mathbf{K e}$ pler's problem of the charge (or mass) motion in the Coulomb (or Newtonian) potential. So, we have

$E_{n}=n \hbar \omega$.

On passing to a rotating coordinate system, we will dispose of the magnetic field, while the quantization of harmonic oscillations is what we can easily do. Meanwhile, the quantization rule $E=n \hbar \omega / 2$ seems to be invalid. But, in fact, everything is true. In a rotating system, all the circles on which in the initial coordinate system the charges revolve with the frequency $\omega$ will turn into the circles or ellipses on which the charges revolve with the frequency $\omega / 2$. The transition $n \rightarrow n-1$ will cause the charge to emit a photon with the frequency $\omega / 2$, as it should be. Let us now slowly turn back to the initial coordinate system and employ Ehrenfest's adiabatic hypothesis (this term was first used by Einstein in 1914): every state, completely definite in terms of the quantum theory, under an adiabatic change of system;s parameters will again turn to a definite state characterized by the same quantum numbers. This means that when we move back to the initial system, all the revolution frequencies $\omega / 2$ will turn to the value $\omega$, and our quantum number $n$ will not change, and, at $n \gg 1$, we will obtain $\boldsymbol{a}$ valid quantization rule of energy levels in a magnetic field ${ }^{18}$.

18 Everything happens in the same way as in the case with the Rayleigh-Lorentz-Einstein pendulum. According to Einstein speaking at I Solvay Conference (1911), “... oscillation energy is still equal to $h \nu$, if it was initially equal to $h \nu$, oscillation energy changes proportional to $\nu$ " [32].

ISSN 2071-0194. Ukr. J. Phys. 2020. Vol. 65, No. 10 
Formula (31), more precisely, the formula $E_{n}=$ $=(n+1 / 2) \hbar \omega$, for arbitrary $n$ was first obtained by Fock in 1928 [66], but back then almost nobody noticed it. In the same year, a relativistic generalization of this formula, $E_{n}=\sqrt{m_{e}^{2} c^{4}+2(n \hbar \omega)^{2}}$, was obtained by Rabi [67] out of Dirac's relativistic equation, which had been just discovered ${ }^{19}$. Nevertheless, Rabi's quantization was taken as some relativistic quirk. Still earlier, in 1923, Wilson [68] had figured out how to generalize Boltzmann's adiabatic invariant $2 \tau \bar{T}$ in the case of charge $e$ revolving in the magnetic field $\mathbf{H}=\operatorname{rot} \mathbf{A}$. We have to make a substitution $(\mathbf{p}, \mathbf{q}$ are the momentum and coordinate of the charge):

$2 \tau \bar{T}=\oint \mathbf{p} d \mathbf{q}=n h \rightarrow \oint\left(\mathbf{p}+\frac{e}{c} \mathbf{A}\right) d \mathbf{q}=n h$.

Formula (32) solved the quasiclassical quantization problem at $n \gg 1$. It turned out later that the substitution in (32) $n \rightarrow n+1 / 2$ yielded an accurate formula to calculate the energy levels of an electron in a static magnetic field and in a quadratic one-dimensional potential well for arbitrary $n$,

$E_{n}=\left(n+\frac{1}{2}\right) \hbar \omega$.

Fock and Rabi solved the problems of the new quantum mechanics, i.e. the Schr'odinger equation and Dirac equation, rather than the old one. Then why had almost nobody noticed Fock's paper [66]? Fock was indifferent about searching for the energy levels of a charge moving in a magnetic field. The following problem also seemed simple to him: a charge is located in a quadratic potential well, which, in its turn, is located in a magnetic field, the energy levels have to be found. While being simple to Fock, it was too complex to most other physicists, its applicability being also obscure. The further discussion will clarify this point.

Fock's classical problem deals with the following equation:

$\ddot{z}-i \omega \dot{z}+\omega_{0}^{2} z=0$.

The motion with definite frequency, $z=R \times$ $\times \exp (-i \Omega t)$, relates to two frequencies $\Omega_{1}$ and $\Omega_{2}$,

\footnotetext{
19 The Rabi formula is an exact formula, but in it, thanks to the magnetic moment of the electron, the "zero oscillations of energy" have disappeared, from $n+1 / 2$ disappeared $1 / 2$.
}

$\Omega_{1,2}=\frac{\omega}{2} \pm \sqrt{\left(\frac{\omega}{2}\right)^{2}+\omega_{0}^{2}}$. What Fock calculated [66] were the energy levels for arbitrary integers $n_{1}$ and $n_{2}$ following from the Schrödinger equation.

$$
\begin{aligned}
& E\left(n_{1}, n_{2}\right)=\left(n_{1}+\frac{1}{2}\right) \hbar\left|\Omega_{1}\right|+\left(n_{2}+\frac{1}{2}\right) \hbar\left|\Omega_{2}\right|= \\
& =\hbar\left[\frac{\omega}{2}\left(n_{1}-n_{2}\right)+\left(n_{1}+n_{2}+1\right) \sqrt{\left(\frac{\omega}{2}\right)^{2}+\omega_{0}^{2}}\right] .
\end{aligned}
$$

At $\omega_{0}=0$ (eliminating the well !), we obtain formula (33) $E_{n_{1}}=\left(n_{1}+\frac{1}{2}\right) \hbar \omega$. At $\omega=0$ (eliminating the magnetic field !) we obtain the formula

$E_{n}=(n+1) \hbar \omega=\left(n_{1}+n_{2}+1\right) \hbar \omega$

i.e. the accurate formula for the quantization in a twodimensional quadratic well. It is easily calculated that all levels in the well are $(n+1)$-fold degenerate. We will see that the energy levels of a charge moving in a magnetic field are also degenerate, but infinity-fold degenerate.

In 1931, Fock's problem was solved by Darwin [69]. He knew nothing about Fock's paper, but he was familiar with paper [70], where Landau proved that the quantum gas of free electrons "possesses orbital diamagnetism, different from zero, ... which is exactly equal to one third of the Pauli spinor paramagnetism" [71]. Darwin's paper was the reaction to Landau's paper. He wrote: "In a recent paper, Landau has shown that, when electrons are moving freely in a magnetic field, they exhibit, in addition to the paramagnetic effect of their spin, a diamagnetic effect due to their motion. This result is rather unexpected, since it is quite contrary to the classical case" ${ }^{20}$. The diamagnetism of free electrons is a specifically quantum effect, but to observe it, the electron motion should be limited by some walls. Landau placed his electrons in a rectangular box, while Darwin replaced vertical walls by a quadratic potential well. This brought him to Fock's problem.

20 Bohr-van Leeuwen theorem, which was not yet forgotten in 1930, stated that "in a uniform magnetic field and in thermal equilibrium, the magnetization of the classical electron gas vanishes identically". Van Leeuwen (1921) [72] proved this theorem independently of Bohr (1911) [73]. A rigorous proof of this theorem is given in paper [74]. 
First, Fock (and later Darwin) obtained formula (35) in the form

$$
E(m, N)=\hbar\left[\frac{\omega}{2} m+(2 N+|m|+1) \sqrt{\left(\frac{\omega}{2}\right)^{2}+\omega_{0}^{2}}\right]
$$

where $m=n_{1}-n_{2}=0, \pm 1, \pm 2 \ldots$ is the azimuthal quantum number, $N=0,1,2, \ldots$ is the radial quantum number. In the absence of the potential well, $\left(\omega_{0}=0\right)$, we get

$E(n(m, N))=\left(n+\frac{1}{2}\right) \hbar \omega=$

$=\left(N+\frac{m+|m|}{2}+\frac{1}{2}\right) \hbar \omega$,

$m=n, n-1, \ldots 0,-1,-2, \ldots-\infty$.

Note that Fock and Darwin were the first who considered "the quantum dot" in a magnetic field, the term "Fock-Darwin quantum dot" is generally accepted in the special literature [75-77]. Quantum dots that are created and studied in laboratories are rightly called artificial atoms, they usually contain many electrons, which are placed at allowed quantum levels, creating shells, if the levels are degenerate. Quantum dots with completely filled shells, as in the case of ordinary atoms, are artificial atoms that are particularly stable.

\subsection{Degeneration of energy levels in a magnetic field and the quantum Hall effect}

According to Darwin, negative $m$ values are responsible both for Landau's diamagnetism of free electrons and for the disappearance of the diamagnetism in the classical limit [69]. In addition, the infinity-fold degeneration of energy levels in a magnetic field (in the absence of a well) due to negative $m$ (38) have caused such fundamental physical consequences that it resulted in two Nobel prizes for the discovery and explanation of the quantum Hall effect (QHE), for integer and fractional cases (K. von Klitzing, 1985; R. Laughlin, H. Stormer, D. Tsui, 1998).

So, what is the essence of the degeneration of energy levels due to negative $m$ ? The equations for the motion of an electric charge in a magnetic field (29), $\ddot{z}-i \omega \dot{z}=0$, contain integrals of motion, crucial for the theory of the quantum Hall effect, as well as coordinates of the circle center, in which the charge is moving, $x_{0}$ and $y_{0}$, or

$$
\begin{aligned}
& z_{0}=x_{0}+i y_{0}=z+\frac{i}{\omega} \dot{z}, \\
& z_{0}^{*}=x_{0}-i y_{0}=z^{*}-\frac{i}{\omega} \dot{z}^{*} .
\end{aligned}
$$

It is clear that arbitrary functions of $z_{0}$ and $z_{0}^{*}$ are also the integrals of motion. Let us calculate the integral of motion

$$
\begin{aligned}
& r_{0}^{2}=\left|z_{0}\right|^{2}=x_{0}^{2}+y_{0}^{2}= \\
& =\underbrace{\dot{z} \dot{z}^{*} / \omega^{2}}+\underbrace{z^{*} z+i\left(z^{*} \dot{z}-\dot{z}^{*} z\right) / \omega}= \\
& =\underbrace{2 E / m_{e} \omega^{2}}-\underbrace{2 M / m_{e} \omega} .
\end{aligned}
$$

According to (40), the integral of motion $r_{0}^{2}$ is expressed through two integrals of motion, the kinetic energy of a charge $E$ and the angular momentum $M$ (relative to the point $z=0$ ). It is worth noting that the relation between $r_{0}^{2}, E$ and $M$ is still present in the quantum case where all quantities become operators [78]. Since $E$ and $M$ are quantized, $r_{0}^{2}$ is also quantized:

$$
\begin{aligned}
& \pi r_{0}^{2}(n, m)=\left(n-m+\frac{1}{2}\right) S_{0}, \\
& m=n, n-1, \ldots 0,-1,-2, \ldots-\infty
\end{aligned}
$$

$S_{0}$ is the area with the magnetic flux passing through which is equal to the flux $\Phi_{0}=h c / e$, i.e. $S_{0}=h c / e H$. The operators $\hat{x}_{0}$ and $\hat{y}_{0}$ do not commute,

$\hat{x}_{0} \hat{y}_{0}-\hat{y}_{0} \hat{x}_{0}=i \frac{S_{0}}{2 \pi}$,

from where the formula (41) follows directly. The physical meaning of the degeneration of energy levels by $m$ consists in the fact that electric charges in the plane permeated by the magnetic field can be localized in a vicinity of any point $\left(x_{0}, y_{0}\right)$, but the localization, according to (42), has quantum limitations $\Delta x_{0} \Delta y_{0} \approx S_{0}$. According to (41), in a circle of the area $\left(n-m+\frac{1}{2}\right) S_{0}$, the charge has $n-m$ possibilities to be placed somewhere, which means that a circle can contain not more than $n-m$ electrons with the fixed direction of the spin (Pauli principle). Thus, we may conclude that the maximum density of electrons in a fixed quantum state on a plane permeated by a 
magnetic field is equal to $1 / S_{0}$, and the charge density is $\rho_{0}=e / S_{0}^{21}$.

Consider now a semiconducting film which is permeated by the magnetic field perpendicular to the film $H_{0}{ }^{22}$. Let the film be maximally packed with electrons which are in the first Landau energy level. If we start moving past the film (along its length) with a velocity of $-V$, in our coordinate system, the electric current $j=\rho_{0} V / \sqrt{1-V^{2} / c^{2}}=e / S_{0} \times$ $\times V / \sqrt{1-V^{2} / c^{2}}$ will appear. In addition, due to the Lorentz transformation, there will appear the electric (Hall's) field $E_{H}=V / c H_{0} / \sqrt{1-V^{2} / c^{2}}$, perpendicular to the velocity $V$ and the magnetic field $H$. So, we obtain

$j=\frac{e}{S_{0}} \frac{V}{\sqrt{1-V^{2} / c^{2}}}=$

$=\frac{e^{2}}{h} \frac{H_{0}}{c} \frac{V}{\sqrt{1-V^{2} / c^{2}}}=\frac{e^{2}}{h} E_{H}=\sigma_{0} E_{H}$.

Note that Eq. (43) is one of three relativistically invariant equations, which describe the motion of electrons in a two-dimensional plane (more specifically, the motion of the electron orbit centers),

$j_{\mu}=\frac{e^{2}}{2 h} \varepsilon_{\mu \nu \rho} F_{\nu \rho}$

$J_{i}=\frac{e^{2}}{h} \varepsilon_{i k} E_{k}, \quad J_{0} \equiv \rho=\frac{e^{2}}{h} H$,

where $\mu, \nu, \rho=0,1,2, i, k, l=1,2, F_{12}=H, F_{i 0}==$ $E_{i}, \varepsilon_{\mu \nu \rho}$ and $\varepsilon_{i k}$ are the Levi-Civita tensors, $\varepsilon_{012}=1$ and $\varepsilon_{12}=1$. If, in a semiconducting film, the electrons occupy $n$ Landau levels, then there appears the quantum Hall effect $j=n \sigma_{0} E_{H}$. In a film with width $L$ and length $L^{*}$, the relation between the total current $I=j L$ and the Hall voltage $U_{H}=E_{H} L$ takes

21 In 1979, a year before QHE was discovered by K. von Klitzing [79], Aharonov and Casher [80] proved the theorem, which is directly related to QHE. The theorem by Aharonov and Casher states that "an electron moving in a plane under the influence of a perpendicular inhomogeneous magnetic field $H(x, y)$ has $N$ ground-energy states, where $N$ is the integral part of the total flux $\Phi$ in units of the flux quantum $\Phi_{0}, N=\left\{\Phi / \Phi_{0}\right\} "$. For more details, see [81].

22 Semiconducting film is required only for the purpose of locating the electrons on a two-dimensional plane. The behaviour of the electrons on this plane is considered not to differ from that of electrons in vacuum. Experiments corroborate this assumption with great accuracy. the form $I=n \sigma_{0} U_{H}$. For the quantization of the Hall resistance $R_{H}(n)$, we obtain the formula

$R_{H}(n)=\frac{h / e^{2}}{n}=\frac{R_{\mathrm{K}}}{n}$,

where $R_{\mathrm{K}}$ is the von Klitzing constant.

In 1980, von Klitzing found the $R_{\mathrm{K}}$ value $R_{\mathrm{K}-1980}=25812.68 \pm 0.08$ Ohm. Using the recommended value of the fine-structure constant (Sommerfeld constant) [47], we obtain

$R_{\mathrm{K}-2019}=\frac{h}{e^{2}}=2 \pi \frac{\hbar c}{e^{2}} \frac{1}{c}=2 \pi \frac{1}{\alpha} \frac{1}{c}=$

$=2 \pi \times 137.035999084(21) \times 29.9792458=$

$=25812.8074452(39)$ Ohm.

When calculating (46), we used the relation Ohm $=$ Volt $/$ Ampere, which allows expressing the speed of light in Ohms, $1 / c \approx 30 \mathrm{Ohm}$, more precisely

Ohm $=\frac{\text { Coulomb } / \text { metr }}{2.99792458^{2} \times 10^{9}} /$ Coulomb $/$ second $=$

$=\frac{1}{29.9792458} \frac{1}{c}$

i.e. $1 / c=29.9792458 \mathrm{Ohm}$ [82]. This relation between the speed of light and Ohms was the one von Klitzing used in 1980. The formula (46) explicitly relates the von Klitzing and Sommerfeld constants. Von Klitzing saw this as the essence of his fundamental discovery, entitling his Nobel-prize-winning publication [79] "New Method for High-Accuracy Determination of the Fine-Structure Constant Based on Quantized Hall Resistance".

Apparently, instead of running past a semiconducting film at a speed of almost $26 \mathrm{~km} / \mathrm{h}$ (we will soon calculate this speed), von Klitzing connected the film of the size $L \times L^{*}=50 \mu \mathrm{m} \times 140 \mu \mathrm{m}$ to a voltage source. The voltage was selected so that the electrons occupying four Landau levels should produce the electric current of $1 \mu \mathrm{A}$ along $L^{*}$. The film was located in a $18 \mathrm{~T}$ magnetic field, and the Hall voltage $U_{H}=6453.17 \pm 0.02 \mathrm{mV}$ was observed. The corresponding Hall field and the velocity of the electron motion were $E_{H}=129 \mathrm{~V} / \mathrm{m}$ and $V=\left(E_{H} / H_{0}\right) c=7.17 \mathrm{~m} / \mathrm{s}$. Since, in von Klitzing's experiments, a static electric current was maintained 
in the film $I=e n V_{n} L H / \Phi_{0}=1 \mu \mathrm{A}$, where $V_{n}$ is the velocity of the electrons with $n$ Landau levels occupied, $n V_{n}=$ const, $n V_{n}=4 V_{4}=4 \times 7.17 \mathrm{~m} / \mathrm{s}$, and we have obtained the formula for the velocity of the electrons $V_{n}=28.68 / n \mathrm{~m} / \mathrm{s}$.

\subsection{Rydberg hydrogen atom in a magnetic field as an anharmonic oscillator and supersymmetric quantum mechanics}

Consider a classical electron with the charge $-e$ and the mass $m_{e}$. Let the electron move around the Coulomb center with the charge $e$ in a plane $(x, y)$, the magnetic field $H$ being directed perpendicularly to it. The equations of the electron motion in the complex plane $z=x+i y,|z|^{2}=r^{2}$,

$\ddot{z}-i \omega \dot{z}+\alpha \frac{z}{|z|^{3}}=0$,

are similar to the equations for the charge motion in a Fock-Darwin quantum dot (34), but they have a singularity in the coordinate origin. In Eq. (48), $\omega=$ $=e H / m_{e} c$ is the cyclotron frequency, $\alpha=2 e^{2} / m_{e}$. In the reference frame $(\tilde{x}, \tilde{y})$ rotating with Larmor's frequency $\omega / 2, \tilde{z}=\tilde{x}+i \tilde{y}=\exp (-i \omega t / 2) z$, we obtain

$\ddot{\tilde{z}}+(\omega / 2)^{2} \tilde{z}+\alpha \frac{\tilde{z}}{|\tilde{z}|^{3}}=0$.

Passing to a new complex variable $w=\xi+i \zeta=$ $=\sqrt{\tilde{z} / 2}=\sqrt{(\tilde{x}+i \tilde{y}) / 2}$ and a new time $\tau, d \tau=$ $=d t /|\tilde{z}|$, we will obtain, instead of (49), the equation of an anharmonic oscillator without singularity,

$w^{\prime \prime}+\Omega^{2} w+3(\omega / 2)^{2}|w|^{4} w=0$,

where $w^{\prime \prime} \equiv d^{2} w / d \tau^{2}, \Omega^{2}$ is the motion integral of Eq. (49),

$\Omega^{2}=-\frac{1}{4}\left(|\dot{\tilde{z}}|^{2}+(\omega / 2)^{2}|\tilde{z}|^{2}-\frac{\alpha}{|\tilde{z}|}\right)$

related to the electron energy $E=-2 m_{e} \Omega^{2}$, whose levels, $E_{n}$, we need to find.

Let us make an assumption whose validity is proved by comparing the calculated and observed energy levels $W_{n}$ [83]: the electronic orbital momentum in a rotating reference frame equals zero. The electron in this system is supposed to move along the axis $\tilde{x}$, $\tilde{x} \equiv r \geq 0$, the corresponding $\xi$ can be both positive and negative. Instead of (50), we have

$\xi^{\prime \prime}+\Omega^{2} \xi+3(\omega / 2)^{2} \xi^{5}=0$.

Let us quantize the motion integral of Eq. (52),

$W=8 m_{e}\left(\xi^{\prime 2}+\Omega^{2} \xi^{2}+(\omega / 2)^{2} \xi^{6}\right)=T+U$.

The respective Bohr-Sommerfeld quantization rule has a form $(n \gg 1)$ :

$$
\begin{aligned}
& \oint \sqrt{2 m_{e}\left(W_{n}-U\right)} d \xi= \\
& =8 \int_{0}^{\xi_{0}} \sqrt{m_{e} W_{n} / 2+2 E m_{e} \xi^{2}-m_{e}^{2} \omega^{2} \xi^{6}} d \xi=n h
\end{aligned}
$$

(at the upper limit of integration, the integrand equals zero). But here arises a problem which is easy to be solved. The fact is that $W$ has an absolutely definite value, $W=2 e^{2}$ which we can clearly see, if we use the relation $\xi^{2}=r / 2, \xi^{\prime 2}=r \dot{r}^{2} / 8$. That is, in order to quantize integral (54), the energy $E$ is to be quantized, which is just what we need:

$$
\begin{aligned}
& \int_{0}^{\xi_{0}} \sqrt{m_{e} e^{2}\left[1+2\left(E_{n} / e^{2}\right) \xi^{2}-m_{e}(\omega / e)^{2} \xi^{6}\right]} d \xi= \\
& =n h / 8 .
\end{aligned}
$$

Passing to a new variable $\lambda=\xi \sqrt{m_{e} e^{2}} / n \hbar$, let us rewrite (55) in the form

$$
\begin{aligned}
& \int_{0}^{\lambda_{0}} \sqrt{1+2\left(E_{n} n^{2} / \hbar \omega_{0}\right) \lambda^{2}-\left(\omega n^{3} / \omega_{0}\right)^{2} \lambda^{6}} d \lambda= \\
& =\pi / 4,
\end{aligned}
$$

where the frequency $\omega_{0}=e^{4} m_{e} / \hbar^{3}=4 \pi \nu_{0}, \nu_{0}$ is the Rydberg constant. Note that the levels $E_{n}$ are characterized by a very small ratio $\omega / \omega_{0}=H \times 4.2543821 \times$ $\times 10^{-10}$, if $H$ is measured in Tesla $(\mathrm{T})$. In the experiments [83], the field $H$ was equal to 2-4 T.

Equation (56) contains an important information on the characteristics of the energy spectrum of Rydberg hydrogen atoms in a magnetic field; it means that $E_{n} n^{2} / \hbar \omega_{0}$ is a function of $\beta=\left(\omega / \omega_{0}\right) n^{3}$, 
$E_{n}(\beta) n^{2}=\hbar \omega_{0} f(\beta)$. This is the well-known empirical scale law [83, 84], and Eq. (57) in the form of $(56)$,

$\int_{0}^{\lambda_{0}} \sqrt{1+2 f(\beta) \lambda^{2}-\beta^{2} \lambda^{6}} d \lambda=\pi / 4$,

allows to calculate the function $f(\beta)$, which agrees perfectly with the experiment [83]. Thus, e.g., it is easy to calculate the value

$\beta_{0}=\left(\frac{4}{\pi} \int_{0}^{1} \sqrt{1-\lambda^{6}} d \lambda\right)^{3}=1.5592627$,

which turns $f(\beta)=0$. It is somewhat more difficult to obtain the formula describing the experimentally derived equidistance of levels (Landau quasilevels) with the difference of $3 \hbar \omega / 2$ in a vicinity of $E_{n}=0$,

$E_{n}=3 \hbar \omega / 2\left(n-33.21704 / H^{1 / 3}\right)$,

where $H$ is measured in Tesla. At $H=1 \mathrm{~T}$, the energy sign changes with the transition from $n=33$ to $n=34$, but, according to (58), $E_{34}-E_{33}=3 \hbar \omega / 2$. If we increase the magnetic field to $H=1.1 \mathrm{~T}$, the energy sign will change with the transition from $n=32$ to $n=33$, i.e. the energy level location is easy to be controlled.

It is clear that Eq. (55) and, hence, (58) are absolutely equivalent to the conventional rule of the Bohr-Sommerfeld quantization [82] (to make the formulae simpler, we will change to atomic units $e=$ $=\hbar=m=1$ )

$$
\begin{aligned}
& \int_{0}^{r_{0}} \sqrt{2 E_{n}+2 / r-(\omega / 2)^{2} r^{2}} d r=n \pi \underset{r \rightleftarrows 2 \xi^{2}}{\rightleftarrows} \\
& \underset{r \rightleftarrows 2 \xi^{2}}{\rightleftarrows} \int_{0}^{\xi_{0}} \sqrt{1+2 E_{n} \xi^{2}-\omega^{2} \xi^{6}} d \xi=n \pi / 4 .
\end{aligned}
$$

What is new in changing to the new variable $\xi$ and the new time $\tau$, except the fact that we have disposed of the singularity $1 / r$ ? The new and significant consequences of our reformulation of the problem are revealed, when we analyze the Schrödinger equation corresponding to an anharmonic oscillator (52),

$\left(-d^{2} / d \xi^{2}-8 E_{n} \xi^{2}+4 \omega^{2} \xi^{6}\right) \psi_{n}=2 \psi_{n}$.
Consider just one example. Equation (58) is an example of the equation

$\left[-d^{2} / d \xi^{2}+\varepsilon d U / d \xi+U^{2}(\xi)\right] \psi_{n}=\lambda_{n} \psi_{n}$

with $U=2 \omega \xi^{3}, \varepsilon_{n}=4 E_{n} / 3 \omega, \lambda_{n}=2$. If $\varepsilon= \pm 1$, and if there exists a quadratically normalized function

$\psi_{0}^{-}(\xi)=\int_{0}^{\xi} e^{-U(\rho)} d \rho$

then, in the a supersymmetric pair of equations [85]

$\left[-d^{2} / d \xi^{2} \pm d U / d \xi+U^{2}(\xi)\right] \psi_{n}^{ \pm}=\lambda_{n}^{ \pm} \psi_{n}^{ \pm}$

all the eigenvalues coincide, except $\lambda_{0}^{+}=0$. That is,

$\lambda_{1}^{+}=0, \lambda_{2}^{+}=\lambda_{1}^{-}, \ldots, \lambda_{n+1}^{+}=\lambda_{n}^{-}, \ldots$

Let us now find the distance between the levels $E_{n}$ in a vicinity of $E_{n}=0$, which, as we already know, are equally spaced. Consider the location of the levels, where the level $E_{n}<0$ and the level $E_{n+1}>0$, and $\left|E_{n}\right|=E_{n+1}$. If $\varepsilon_{n}=4 E_{n} / 3 \omega=-1$, and $\varepsilon_{n+1}=$ $=4 E_{n+1} / 3 \omega=1$, the corresponding equations (63) turn into a supersymmetric pair of equations with the same eigenvalue $\lambda_{n}^{-}=\lambda_{n-1}^{+}=2$, and $E_{n+1}-E_{n}=$ $=3 \omega / 2 \equiv 3 \hbar \omega / 2$, as it should be.

We have just discussed a simplified but significant case of a flat Rydberg hydrogen atom in a magnetic field using the simplified regularization of Kepler's problem. This case was also considered in papers $[86,87]$. The generalization of the spinor regularization for a classical and quantum three-dimensional hydrogen atom in static and homogeneous electric and magnetic fields was analyzed in [88] in detail. Paper [89] reveals the close association between the quantum mechanics of the charge motion in a magnetic field and the supersymmetric quantum mechanics.

\section{Conclusions}

When Newton was writing his PRINCIPIA, he was aided by a junior assistant, who was also a secretary, a copyist and, more importantly, a good nanny, whom Newton needed badly at that time - it was Humphrey Newton. In his memoirs, Humphrey Newton recollected [26] that, within five years, he had seen only once that Newton Senior had laughed - 
when asked why to study Euclid. A really funny question, indeed. Apparently, a lot of things that are just as important as Euclid's mathematics have appeared for 2300 years since the time, when Euclid lived. The idea that one should study not only Euclid (and physicists should study not only Landau and Lifshitz) is probably the main conclusion of this paper. Unfortunately, even outstanding people can overlook important things that could be of interest and useful for them. The spinor regularization of the Kepler problem, for example, escaped Arnold's attention [23], who was so perfect at binding square roots from Kepler ellipses and Zhukovsky transformation. Using the Zhukovsky transformation, our paper explains that finding a square root of the Kepler ellipse and employing the Kepler eccentric anomaly instead of the conventional time is what makes up the spinor regularization of the Kepler problem.The spinor regularization is generalized to non-ellipic orbits. This is used to analyze the energy spectra of Rydberg hydrogen atoms in a magnetic field. Some of the important characteristics of these spectra are accounted for by supersymmetric quantum mechanics. An interesting aspect of the classical problem of the charge motion in a static homogeneous magnetic field has been revealed. This problem is mathematically equivalent to the Kepler problem on the mass motion in the Newtonian potential. The history of how Kharkiv radio-astronomers were searching for the recombination radiolines of Rydberg carbon atoms using radio-telescope has been given a detailed description.

The author expresses his sincere gratitude to O.S. Bakai for his continued deep interest in this work and great help in writing this paper.

\section{APPENDIX \\ On major innovations in the SI system and some related problems with new Ohms and the speed of light}

It is worth noting that, quite recently in May 2019, an important event took place: a new international SI system [90], radically changed and enhanced, came into force, which was proposed by the International Bureau of Weights and Measures (Bureau International des Poids et Mesures, BIPM). As it was proclaimed in 1875, when Paris Metre Convention ${ }^{23}$,

${ }^{23}$ From now on, Ukraine as a member state of Paris Metre Convention (since May 2018) is to follow the new SI system. was founded, the new system has also been adopted $A$ TOUS LES TEMPS, A TOUS LES PEOPLES (for all times, for all nations). According to the new updates, from now on the Planck constant

$h=6.62607015 \times 10^{-34} \mathrm{~B} \cdot \mathrm{A} \cdot \mathrm{c}^{2}$

and the electron's electric charge

$e=1.602176634 \times 10^{-19} \mathrm{~A} \cdot \mathrm{c}$

are exactly fixed physical constants, as well as the speed of light

$c=299792458 \mathrm{~m} \cdot \mathrm{c}^{-1}$,

which became a physical constant back in 1983. Von Klitzing constant $R_{\mathrm{K}}$, which is responsible for the superaccurate value of Ohm, as well as the Josephson ${ }^{24}$ constant $K_{\mathrm{J}}$, which is responsible for the superaccurate value of Volt, henceforth and forever can be calculated using a simple arithmetic,

$R_{\mathrm{K}}=h / e^{2}=25812.8074593045 \ldots \mathrm{Ohm}$,

$K_{\mathrm{J}}=2 e / h=483597.848416984 \ldots \mathrm{GHz} / \mathrm{V}$

(three dots in these expressions mean the possibility of calculation of the given numbers with arbitrary accuracy).

These innovations have also caused some inconveniences (probably, temporary ones, if the exact value of the fine structure could be found). If we carefully examine $R_{\mathrm{K}-2019}$ (46) and the new $R_{\mathrm{K}}$, one can notice that the tenth digits in $R_{\mathrm{K}-2019}$ (measured) and in the new $R_{\mathrm{K}}$ (calculated) are different. It happened because the new $R_{\mathrm{K}}$ denotes the new $\mathrm{Ohm}$, $\mathrm{Ohm}_{2019}$, which is slightly less than the older one, which we will designate as $\mathrm{Ohm}_{1948}$, as it was closely (and exactly) related to the new definition of Ampere, which came into effect in 1948 ,

$\mathrm{Ohm}_{1948}=1.00000000055(15) \mathrm{Ohm}_{2019}$.

Despite the fact that $c, h$ and $e$ are exactly defined fundamental physical constants, we cannot calculate exactly the fine structure constant $e^{2} / \hbar c$, as now we do not know how the exact fundamental constant $c$ relates to the new, exactly defined $\mathrm{Ohm}_{2019}$. If we calculate $1 / c$ as

$R_{\mathrm{K}} / 2 \pi \alpha_{2019}$

then

$1 / c=29,9792458163(46) \mathrm{Ohm}_{2019}$.

There is another victim of the updated SI system - an important quantity, exact earlier, - the characteristic vacuum impedance [92], which is equal to $4 \pi / c$ and generally required in Ohms, because it is used to express such practical quantities as the impedances of different electromagnetic wave transmission lines, for example, the impedance of an ordinary TV cable. Of course, the relation between $1 / c$ and the new $\mathrm{Ohm}$ will

${ }^{24}$ On Josephson and on the Nobel prizes related to precision problems, see [91].

ISSN 2071-0194. Ukr. J. Phys. 2020. Vol. 65, No. 10 
become exact as soon as the exact value of the fine structure constant is determined. That is, if it exists at all, rather than changes over time, which is quite possible [93].

1. G. Chr. Lichtenberg. Vermischte Schriften, Band 2 (Dieterich, 1801).

2. P. Nowak. Hodowanie Troglodytów (Kronos, 2014).

3. V.I. Arnold. Do You Need Mathematics at School? (MCNMO, 2004) (in Russian).

4. N.M. Karamzin History of the Russian State, Vol. 1 (Terra, 1998) (in Russian).

5. Galileo Galilei. Opere, Volume II (Nicolo Bettoni e Comp, 1832).

6. Galileo Galilei. Il Saggiatore (Roma, 1623).

7. Yu.A. Bely. Tycho Brahe (Nauka, 1982) (in Russian)

8. Yu.A. Bely. Johannes Kepler(Nauka, 1971) (in Russian).

9. V.E. Thoren, J.R. Christianson. The Lord of Uraniborg: A Biography of Tycho Brahe (Cambridge Univ. Press, 1990) [ISBN: 978-0-521-35158-4].

10. M. Caspar. Kepler (Dover, 1993) [ISBN: 0-486-67605-6].

11. V. Steklov. Galilei (GIZ, 1923) (in Russian).

12. A. Koestler. The Sleepwalkers: A History of Man's Changing Vision of the Universe (Hutchinson, 1959) [ISBN: 014-019246-8].

13. J. Keplerus. Mysterivm Cosmographicvm (Gruppenbachius, 1596).

14. J. Keplerus Astronomia Nova AITIO $\Lambda$ ОГТТО (Vögelin, 1609).

15. J. Keplerus. Ioannis Keppleri Harmonices Mvndi (Lincii, 1619)

16. F. Arago. Biographies of Famous Astronomers, Physicists, and Geometers. Vol. 1 (RCD, 2000) (in Russian).

17. A. Einstein. Mein Weltbild (Ullstein Taschenbuch, 2005) [ISBN: 978-3-548-36728-6].

18. W. Pauli, C. Jung. Atom and Archetype: The Pauli/ Jung Letters 1932-1958 (Princeton Univ. Press, 2001) [ISBN: 9780415120784].

19. A. Pais. The Genius of Science: A Portrait Gallery (Oxford Univ. Press, 2000) [ISBN: 9780198506140].

20. W. Pauli. The influence of archetypal ideas on the scientific theories of Kepler. In: W. Pauli. Writings On Physics And Philosophy (Springer, 1994) [ISBN: 978-3-662-02994-7].

21. M.M. Nieto. The Titius-Bode Law of Planetary Distances: Its History and Theory (Pergamon Press, 1972) [ISBN: 978-1483126944].

22. M.A. Blagg. On a suggested substitute for Bode's Law. Mon. Not. R. Astron. Soc. 73, 414 (1913).

23. V.I. Arnold. Huygens and Barrow, Newton and Hooke (Birkhäuser, 2012) [ISBN: 978-3-0348-9129-5].

24. I. Newton. Philosophiae Naturalis Principia Mathematica (Londini, 1687).

25. https://solarsystem.nasa.gov.

26. P. Ackroyd. Newton (Nan A. Talese, 2008) [ISBN: 9780385507998]

ISSN 2071-0194. Ukr. J. Phys. 2020. Vol. 65, No. 10
27. N.A. Beliaev, K.I. Churiumov. Halley's Comet and Its Observation (Nauka, 1985) (in Russian).

28. T. Levi-Civita. Trajettorie singolari ed urti nel problema ristretto dei tre carpi. Ann. Math. 9, 1 (1903).

29. P. Kustaanheimo. Spinor regularization of Kepler motion. Ann. Univ. Turkuensis A. 73, 3 (1964).

30. N. Joukowsky. Über die Konturen der Tragflächen der Drachenflieger. Z. Flugtechnik 1, 281 (1910), 3, 81 (1912).

31. E. Stiefel, G. Scheifele. Linear and Regular Celestial Mechanics (Springer, 1971) [ISBN: 978-3-642-65029-1].

32. A.S. Bakai, Yu.P. Stepanovsky. Adiabatic Invariants (Naukova Dumka, 1981) (in Russian).

33. I.S. Shklovsky. Mind, Life, Universe (Janus-K, 1996) (in Russian).

34. G.E. Gorelik. The Soviet Life of Lev Landau through the Eyes of Eyewitnesses (Vagrius, 2009) [ISBN: 978-5-98264035-2] (in Russian).

35. L.D. Landau, E.M. Lifshits. Theoretical physics. Quantum mechanics. Part 1. Nonrelativistic theory (GITTL, 1948) (in Russian).

36. E. Fermi. Uber die magnetischen momente der atomkerne Zeits. für Physik 60, 320 (1930).

37. E. Fermi. Magnetic moments of atomic nuclei. Nature 125, 16 (1930).

38. C.G. Darwin. The wave equations of the electron. Proc Roy. Soc. Lond. A118, 654 (1928).

39. E. Fermi, E. Segrè. Zur theorie der hyperfeinstrukturen. Zeits. für Physik 82, 729 (1933).

40. I. Pomeranchuk, Y. Smorodinsky. On the energy levels of systems with $Z>137$. J. Phys. USSR 9, 97 (1945).

41. L. Fermi. Atomi in famiglia (Mondadori, 1954).

42. J.E. Nafe, E.B. Nelson, I.I. Rabi. The hyperfine structure of atomic hydrogen and deuterium. Phys. Rev. 71, 914 (1947).

43. H.M. Foley, P. Kusch. On the intrinsic moment of the electron. Phys. Rev. 73, 412 (1948).

44. J. Schwinger. On quantum electrodynamics and the magnetic moment of the electron. Phys. Rev. 73, 416 (1948).

45. D. Hanneke, S. Fogwell, G. Gabrielse. New measurement of the electron magnetic moment and the fine structure constant. Phys. Rev. Lett. 100, 120801 (2008).

46. T. Aoyama, M. Hayakawa, T. Kinoshita, M. Nio. Revised value of the eighth-order electron $g-2$. Phys. Rev. Lett. 99, 110406 (2007).

47. http://pdg.lbl.gov/2019/reviews/rpp2019-rev-physconstants.pdf.

48. S.G. Karshenboim. Precision physics of simple Atoms: QED tests, nuclear structure and fundamental constants. Phys. Rept. 422, 1 (2005).

49. E.U. Condon, G.H. Shortley The Theory of Atomic Spectra (Cambridge Univ. Press, 1935).

50. I.B. Khriplovich, A.I. Milstein, S.S. Petrosyan. Nuclear structure corrections to deuterium hyperfine splitting. Phys. Lett. B 366, 13 (1996). 
51. I.S. Shklovsky. Cosmic Radio Waves (Harvard Univ. Press, 1960).

52. W.W. Holloway, Jr. and R. Novick. Determination of the hyperfine structure of atomic nitrogen by optical rientation. Phys. Rev. Lett. 1, 367 (1958).

53. J.D. Kraus. Radio Astronomy (Cygnus-Quasar Books, 2005).

54. A.A. Konovalenko, L.G. Sodin. Neutral ${ }^{14} N$ in the interstellar medium. Nature 283, 360 (1980).

55. D.H. Blake, R.M. Crutcher, W.D. Watson. Identification of the anomalous 26.131- $\mathrm{MHz}$ nitrogen line observed towards Cas A. Nature 287, 707 (1980).

56. A.A. Konovalenko, L.G. Sodin. The $26.13 \mathrm{MHz}$ absorption line in the direction of Cassiopeia A. Nature 294, 135 (1981).

57. V.M. Kontorovich, S.Ya. Braude. Radio Waves Tell about the Universe (Fizmatlit, 2011) (in Russian).

58. B.P. Ryabov. Jovian decametric emission. Multiscale dynamic spectra. Radio Phys. Radio Astron. 6 (1), 103 (2001).

59. Academician S. Ya. Braude in the memoirs of contemporaries (Radio Astronomical Institute of the NAS of Ukraine, 2005) (in Russian).

60. A.A. Konovalenko. I.S. Shklovsky and low-frequency radio astronomy. Radio Phys. Radio Astron. 22 (1), 7 (2017).

61. A. Konovalenko, L. Sodin, V. Zakharenko, et al. The modern radio astronomy network in Ukraine: UTR-2, URAN and GURT. Exper. Astron. 4211 (2016).

62. M.A. Gordon, R.L. Sorochenko. Radio Recombination Lines (Springer, 2009).

63. P.S. Ehrenfest - A.F. Ioffe: Scientific Correspondence (1907-1933) (Nauka, 1990) (in Russian).

64. L.D. Landau, E.M. Lifshitz. Mechanics (Nauka, 1988) [ISBN: 5-02-013850-9] (in Russian).

65. L.D. Landau, E.M. Lifshits. Field Theory (Nauka, 1988) [ISBN: 5-02-014420-7] (in Russian).

66 . V. Fock. Bemerkung zur quantelung des harmonischen oszillators im magnetfeld. Zeits. für Physik 47, 446 (1928).

67. I.I. Rabi. Das freie Elektron im homogenen magnetfeld nach der diracschen theorie. Zeits. für Physik 49, 507 (1928).

68. W. Wilson. The quantum theory and electromagnetic phenomena. Proc. Roy. Soc. London A 102, 478 (1923).

69. C.G. Darwin. The diamagnetism of the Free electron. Math. Proc. Cambr. Phil. Soc. 27, 86 (1931).

70. L. Landau. Diamagnetismus der metalle. Zeits. für Physik 64, 629 (1930).

71. W. Pauli. Über gasentartung und paramagnetismus, Zeits. für Physik 41, 81 (1927).

72. H.-J. van Leeuwen. Problèmes de la théorie électronique du magnétisme. J. Phys. Radium 2 (12), 361 (1921).

73. N. Bohr. Collected Works, Vol. 1, Early Works (19051911) (Elsevier, 1972).

74. Baptiste Savoie. A rigorous proof of the Bohr-van Leeuwen theorem in the semiclassical limit. Rev. Math. Phys. 27, 1550019 (2015).

75. L. Kouwenhoven, C. Marcus. Quantum dots. Physics World 11(6), 35 (1998).

76. D. Bimberg, M. Grundmann, N.N. Ledentsov. Quantum Dot Heterostructures (Wiley, 1999) [ISBN: 978-0-47197388-1].

77. E. Drigho-Filho, S. Kuru, J. Negro, L.M. Nieto. Superintegrability of the Fock-Darwin system. Ann. Phys. 383, 101 (2017).

78. V.M. Galitsky, B.M. Karnakov, V.I. Kogan. Problems in Quantum Mechanics (Nauka, 1992) (in Russian).

79. K. von Klitzing, G. Dorda, M. Pepper. New method for high-accuracy determination of the fine-structure constant based on quantized Hall resistance. Phys. Rev. Lett. 45, 494 (1980).

80. Y. Aharonov, A. Casher. Ground state of a spin- $1 / 2$ charged particle in a two-dimensional magnetic field. Phys. Rev. A 19, 2461 (1979).

81. Yu.P. Stepanovsky. Fractional quantum Hall effect. Electromagn. Phenom. 1, 427 (1998).

82. E.M. Purcell. Electricity and Magnetism (Cambridge Univ. Press, 2011) [ISBN: 978-1-107-01360-5].

83. R.J. Fonck, F.L. Roesler, D.H. Tracy. Comparison of atomic quasi-Landau spectrum with semiclassical strongfield-mixing models. Phys. Rev. A 21, 861 (1980).

84. S. Feneuille. Semiempirical scaling laws for adiabatic energy levels of highly excited hydrogen atom in high magnetic fields. Phys. Rev. A 26, 673 (1982).

85. F. Cooper, A. Khare, U. Sukhatme. Supersymmetry in Quantum Mechanics (World Scientific, 2001) [ISBN: 978981-238-650-2].

86. Yu.P. Stepanovsky. Hydrogen atom in a magnetic field, super WKB quantization and Majorana's equation. In: Problems of Theoretical Physics (Naukova Dumka, 1991) (in Russian).

87. A. Krot'ko, Yu. Stepanovsky. Highly excited two-dimensional hydrogen atom in magnetic field. Visnyk Lviv Univ. Ser. Fiz. 39, 54 (2006).

88. Yu. P. Stepanovsky. A hydrogen atom in an external field as an anharmonic oscillator. Ukr. J. Phys. 32, 1316 (1987).

89. I.E. Ovcharenko, Yu. P. Stepanovsky, On some properties of 2-D Weyl equation for charged massless spin 1/2 particle. Probl. Atom. Sci. Techn. 3 (1), 56 (2007).

90. Le Système international d'unités (SI)/The International System of Units (SI) (BIPM, 2019).

91. Yu. P. Stepanovsky. "Nobel" physical phenomena and some concepts of modern physics. In: Fundamental Problems of Precision Theory (Nauka, 2001) [ISBN: 5-02-024947-5].

92. S.J. Orfanidis. Electromagnetic Waves and Antennas (Rutgers Univ., 2016).

93. C.J.A.P. Martins. The status of varying constants: a review of the physics, searches and implications. Rep. Prog. Phys. 80, 126902 (2017).

Received 08.07.20.

Translated from Ukrainian by G.M. Lavrova

ISSN 2071-0194. Ukr. J. Phys. 2020. Vol. 65, No. 10 


\section{Ю.П. Степановсъкий}

КВАДРАТНІ КОРЕНІ ІЗ ЕЛІПСІВ

КЕПЛЕРА, ЕЛЕКТРОНИ ТА РІДБЕРІІВСЬКІ

АТОМИ У МАГНІТНОМУ ПОЛІ

$\mathrm{P}$ е $з$ ю м е

Сміливі ідеї юного Кеплера щодо будови Сонячної системи застосовуються до аналізу планетних відстаней в екзопланетній системі HD 10180. За допомогою перетворень Жу- ковського роз'яснюється суть спінорної регуляризації задачі Кеплера, як добування квадратного кореня з еліпса і використання кеплеровської ексцентричної аномалії замість звичайного часу. Розглядаються досягнення харківських радіоастрономів у пошуках рекомбінаційних радіоліній рідбергівських атомів вуглецю на радіотелескопі УТР2. Узагальнення спінорної регуляризації задачі Кеплера на нееліптичні орбіти використовується для аналізу енергетичних спектрів рідбергівських атомів водню в магнітному полі. 